Evolução de espécies: modelos estocásticos para seleção natural por meio de
competição e mutação

\author{
Carolina Bueno Grejo
}

TESE APRESENTADA

$\mathrm{AO}$

Instituto de Matemática e Estatística

DA

Universidade de São Paulo

PARA

OBTENÇÃO DO TÍTULO

$\mathrm{DE}$

Doutor em CiÊnCIAS

Programa: Estatística

Orientador: Prof. Dr. Fábio Prates Machado

Durante o desenvolvimento deste trabalho a autora recebeu auxílio financeiro da CAPES/CNPq

São Paulo, 02 de Setembro de 2016 


\section{Evolução de espécies: modelos estocásticos para seleção natural por meio de competição e mutação}

Esta versão da tese contém as correções e alterações sugeridas pela Comissão Julgadora durante a defesa da versão original do trabalho, realizada em 02/09/2016. Uma cópia da versão original está disponível no Instituto de Matemática e Estatística da Universidade de São Paulo.

Comissão Julgadora:

- Prof. Dr. Fabio Prates Machado (orientador) - IME-USP

- Prof. Dr. Valdivino Vargas Júnior - IME - USP (Pós Doc)

- Prof. Dr. Pablo Martin Rodriguez - ICMC - USP

- Prof. Dr. Cristian Favio Coletti - CMCC UFABC

- Prof. Dr. Fabio Marcellus Lima Sá Makiyama Lopes - Universidade do Chile 
Dedico ao Hectore Secolin 


\section{Agradecimentos}

A realização deste trabalho não poderia ter ocorrido sem a participação de algumas pessoas, às quais dirijo os meus sinceros agradecimentos.

Ao meu orientador Prof. Dr. Fábio Prates Machado pela confiança e parceria ao longo do desenvolvimento deste trabalho.

À minha mãe Maria Inês Bueno Grejo, minha irmã Camila, meu cunhado Pablo, às pequenas Alice e Ísis e à minha tia Maria de Lourdes, por todo apoio e incentivo.

Ao meu eterno amigo e companheiro Hectore Secolin por ser o meu ponto de apoio em todos os momentos e pelo total empenho em sempre incentivar a minha carreira acadêmica.

Aos meus amigos Ana Elisa, Michele, Cintia, Douglas, Amanda, Natália, Christiana e Camila, que apesar de distantes sempre se fizeram presentes.

Aos amigos Alejandro Roldán Correa e Iara Frondana por todas as dicussões, nem sempre matemáticas, ao longo destes anos.

Aos professores Cristian Favio Coletti, Fábio Marcellus Lima Sá Makiyama Lopes, Pablo Martin Rodriguez e Valdivino Vargas Junior pelas importantes contribuições feitas para este trabalho.

Aos professores do programa de Pós-Graduação em Estatística da Universidade de São Paulo que muito contribuíram para minha formação acadêmica.

À Universidade de São Paulo, a Coordenação de Aperfeiçoamento de Pessoal de Nível Superior (Capes) e ao Conselho Nacional de Desenvolvimento Científico e Tecnológico (CNPq) pelo apoio financeiro destinado a elaboração deste trabalho. 


\section{Resumo}

Bueno Grejo, C. Evolução de espécies: modelos estocásticos para seleção natural por meio de competição e mutação. 2016. 61 f. Tese (Doutorado) - Instituto de Matemática e Estatística, Universidade de São Paulo, São Paulo, 2016.

Apresentamos nesse trabalho uma proposta de dois modelos aleatórios para a evolução de espécies. O primeiro para a evolução via seleção natural por meio de competição entre espécies e o segundo por mutação. No primeiro caso consideramos uma versão do modelo proposto por Guiol, Machado e Schinazi no caso subcrítico. Para tal modelo derivamos a distribuição da aptidão de sobrevivência ao meio do indivíduo mais forte ao longo de uma excursão ao zero. Para o segundo modelo proposto, consideramos a mutação como uma estratégia para sobrevivência ao meio e provamos condições para sobrevivência e morte quando o modelo evolui sobre a árvore binária orientada com raiz, provando a existência de transição de fase no modelo.

Palavras-chave: evolução de espécies, modelagem estocástica, transição de fase. 


\section{Abstract}

Grejo, C. B. Evolution of species : Stochastic models for natural selection through competition and mutation . 2016. 61 f. Tese (Doutorado) - Instituto de Matemática e Estatística, Universidade de São Paulo, São Paulo, 2016.

Here we present a proposal for two random models for the evolution of species. To the first one we consider the evolution via natural selection through competition between species and to the second one by mutation. In the first case we consider a version of the model proposed by Guiol, Machado and Schinazi at the subcritical case. For this model we derive the distribution of fitness of the strongest individual along a excursion to zero. For the second one we consider mutation as a strategy for survival and we prove conditions for death and survival when the model evolves on the binary tree with root oriented and we prove the existence of phase transitions in the model.

Keywords: evolution of species, stochastic modeling, phase transition. 


\section{Sumário}

$\begin{array}{ll}\text { Lista de Figuras } & \text { ix }\end{array}$

1 Introdução 1

2 O modelo Guiol-Machado-Schinazi com dinâmica de competição entre espécies $\quad 3$

2.1 O modelo Guiol-Machado-Schinazi . . . . . . . . . . . . . . . . . . 3

2.2 O modelo GMS com competição entre espécies . . . . . . . . . . . . . . . . 4

3 Processo estocástico para evolução por meio de mutação 9

3.1 Processo de infecção para o caso determinístico . . . . . . . . . . . . . . . 10

3.1.1 Análise de sobrevivência considerando um ramo fixo . . . . . . . . . . 11

3.1.2 Análise de extinção e sobrevivência para o processo na árvore ... . 19

3.2 Processo de infecção para o caso aleatório . . . . . . . . . . . . . . 27

3.2.1 Análise de sobrevivência e extinção considerando um ramo fixo . . . . 27

3.2.2 Análise de extinção para o processo de infecção na árvore . . . . . . . 31

$\begin{array}{lll}\text { A } & \text { Passeio aleatório simples (PAS) em } \mathbb{Z} & 37\end{array}$

A.1 Propriedade de transiência e recorrência do passeio aleatório simples . . . . . 38

B Processo de ramificação 41

C Equações, proposição e lema auxiliares $\quad 45$

C.1 Equação 5.2.13.9 Prudnikov et al. (2002) . . . . . . . . . . . . . . . . . 45

C.2 Equação 7.512.11 Gradshteyn e Ryzhik (2007) . . . . . . . . . . . . . . 45

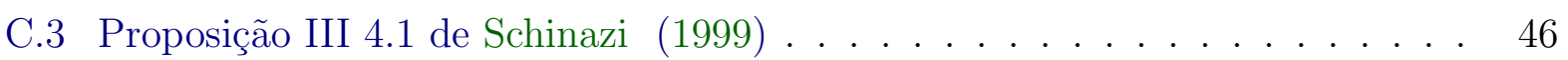

C.4 Lema auxiliar . . . . . . . . . . . . . . . . . . . . . . 46

$\begin{array}{ll}\text { Referências Bibliográficas } & 47\end{array}$ 


\section{Lista de Figuras}

2.1 Histograma do fitness da espécie mais forte no GMS(1) e GMS(10), respectivamente, após 200.000 nascimentos e mortes para $p=1 / 4$. . . . . . . . 4

3.1 Gráfico das funções $\lambda=\frac{1}{r}$ e $\lambda=\frac{1+\sqrt{2}}{r}$, respectivamente. . . . . . . . . . . . 14

3.2 Gráfico das funções $\lambda=\frac{1}{r}, \lambda=\frac{1+\sqrt{5}}{2 r}$ e $\lambda=\frac{1+\sqrt{2}}{r}$, respectivamente. . . . . . . 15

3.3 Representação da numeração de cada vértice da árvore binária até a origem do terceiro tipo. . . . . . . . . . . . . . . . . . . 20

3.4 Gráfico das funções $g(\lambda r)=\frac{1}{4}\left(1-6 \lambda r-\sqrt{1+12 \lambda r+20(\lambda r)^{2}}\right)$ e $h(\lambda r)=$ $\frac{1}{4}\left(1-6 \lambda r-\sqrt{1+12 \lambda r+20(\lambda r)^{2}}\right), \operatorname{com} 0<\lambda r<1,5 \ldots \ldots 26$

3.5 Representação das formas de como uma partícula pode ocupar a posição $\bar{n}=$ $(1,1)$, uma vez que a partícula $\bar{n}=1$ já nasceu. Os traços em vermelho representam a primeira mutação ocorrida. . . . . . . . . . . . . . 28

3.6 Gráfico da função $f(x)=-2 x^{2}+2 x+2$, com $-1<x<2$. . . . . . . . . . 30

3.7 Gráfico das funções $f(p)=1-p-\sqrt{p^{2}-2 p+2}$ e $g(p)=1-p+\sqrt{p^{2}-2 p+2}$, com $0 \leq p \leq 1 \ldots \ldots \ldots \ldots \ldots \ldots$

3.8 Representação das formas de como uma partícula pode ocupar a posição $\bar{n}=$ $(1,2)$. Os traços em vermelho representam a primeira mutação ocorrida e os em azul a segunda. . . . . . . . . . . . . . . . . . . 32

3.9 Gráfico das funções $f(r)=\frac{3-2 \sqrt{2}}{r}$ e $g(r)=\frac{1+\sqrt{5}}{2 r}$, com $0 \leq r \leq 1 . \ldots . . . \quad 35$

B.1 Função geradora do número de filhos $(\gamma)$ nos casos em que $m \leq 1$ e $m>1$, respectivamente. 


\section{Capítulo 1}

\section{Introdução}

Apresentamos neste trabalho dois modelos aleatórios, e suas respectivas variações, que modelam a evolução de organismos vivos. O primeiro modelo considera a evolução por meio do processo denominado competição, ou seja, os indivíduos (ou organismos) menos adaptados ao ambiente são eliminados, prevalecendo os mais bem adaptados. Para o estudo matemático de tal processo evolutivo consideramos o modelo apresentado por Guiol et al. (2011) no caso subcrítico. Neste contexto temos que o sistema se torna vazio infinitas vezes. Como tal modelagem descreve o processo evolutivo por seleção natural, podemos dizer que o último indivíduo presente, antes do sistema se tornar vazio, foi o mais bem adaptado dentre todos os indivíduos que surgiram ao longo do processo.

A segunda modelagem proposta leva em consideração o processo evolutivo por meio de mutação e considera a atuação do sistema imunológico no combate ao agente infeccioso (patógeno). Para entendermos o comportamento de nosso organismo frente a tal agente, precisamos entender o funcionamento do nosso sistema imunológico. Para tal, usamos as definições apresentadas em Abbas et al. (2005).

Podemos definir, de modo abrangente, que imunidade é a reação do nosso organismo frente a presença de substâncias estranhas. Nosso sistema imunológico é composto por um conjunto de células e moléculas responsáveis pela proteção contra as doenças (ou seja, pela imunidade) e a resposta imunológica é a resposta coletiva e coordenada contra as substâncias estranhas presentes no organismo. Possuímos dois tipos de imunidade, uma denominada imunidade natural (também chamada de inata ou nativa) e a imunidade adaptativa (ou adquirida).

A imunidade natural é a que possuímos independentemente de termos tido uma infecção. Assim que a infecção é detectada, ela reage rapidamente. Sua reação é somente contra microrganismos, não apresentando reação contra substâncias que não causem infecções, e sua resposta é realizada praticamente do mesmo modo para sucessivas infecções. Este fato faz com que a imunidade natural seja a responsável pela defesa inicial de nosso organismo contra os microrganismos.

A imunidade adaptativa, que é a que consideraremos em nosso modelo, ao contrário da 
imunidade natural, é estimulada pela exposição a agentes infecciosos e tem tanto sua intensidade quanto sua capacidade de defesa aumentadas se o organismo é exposto novamente a um microrganismo em particular. Uma importante característica da imunidade adaptativa é a especificidade para distinguir os diferentes antígenos presentes no organismo. Quando detectado um antígeno novo, os linfócitos T e B são acionados, por serem responsáveis pela criação de novos anticorpos, capazes de combaterem o agente estranho. Para o hospedeiro, a importância da imunidade adaptativa está no fato que, apesar da imunidade natural realizar uma defesa eficaz contra as infecções, muitos microrganismos patogênicos desenvolvem uma resistência ou então sofrem mutação, a fim de gerar patógenos desconhecidos sendo, então, necessária a atuação da imunidade adaptativa.

Assim, no cenário do segundo modelo, dizer que surgiu um novo tipo de indivíduo no sistema é o mesmo que dizer que ocorreu uma mutação genética. Vamos considerar que todos os indivíduos podem mutar, no entanto cada tipo de indivíduo irá gerar o mesmo tipo de mutação e a cada mutação de tipos é gerado um patógeno desconhecido para o sistema imunológico.

No contexto de tudo o que foi apresentado, o presente texto está dividido do seguinte modo: no capítulo 2 apresentamos o modelo proposto por Guiol et al. (2011) e consideramos o caso subcrítico, ou seja, quando o sistema se torna vazio infinitas vezes. Derivamos a distribuição do fitness do indivíduo mais forte, que é dado pela função Hipergeometrica de Gauss. Os resultados apresentados nesse capítulo são referentes ao trabalho publicado por Grejo et al. (2016).

No capítulo 3 unimos as ideias presentes em Guiol et al. (2011) e em Schinazi e Schweinsberg (2008) para propomos uma modelagem para a evolução por meio de mutação, considerando a atuação do sistema imunológico no combate de tal infecção. O modelo apresentado evolui sobre a árvore binária orientada com raiz e consideramos duas dinâmicas diferentes para a evolução do processo infeccioso, uma determinística e a outra aleatória. Para o modelo determinístico apresentamos condições para a sobrevivência e extinção tanto do processo em um ramo fixado na árvore, quanto do processo como um todo. Considerando o modelo aleatório provamos a existência de transição de fase ao considerar a evolução do processo em um ramo fixo, já para a evolução do modelo em toda a árvore verificamos a influência da aleatoriedade na extinção do processo.

Por fim, apresentamos um apêndice contendo os processos aleatórios clássicos utilizados ao longo do texto, bem como os seus resultados, além de equações e proposições que nos auxiliaram em algumas provas ao longo de todo o texto. 


\section{Capítulo 2}

\section{O modelo Guiol-Machado-Schinazi com dinâmica de competição entre espécies}

Guiol, Machado e Schinazi propuseram em Guiol et al. (2011) um modelo aleatório para a evolução de espécies e o consideramos no caso subcrítico. Nessa situação, o sistema se torna vazio infinitas vezes e, então, dizemos que a última espécie a ser extinta será a que tiver uma maior adaptação ao meio, ou seja, o maior fitness. Nessas condições, apresentamos a distribuição do fitness do indivíduo mais bem adaptado e um fato interessante é a de que tal distribuição é apresentada por meio da função hipergeométrica de Gauss.

Os resultados que seguem são parte do trabalho publicado por Grejo et al. (2016).

\section{$2.1 \quad$ O modelo Guiol-Machado-Schinazi}

O modelo apresentado nessa seção, denominado GMS, foi estudado primeiramente por Guiol et al. (2011) e algumas variações interessantes do modelo foram estudadas por Guiol et al. (2013), Ben-Ari et al. (2011) e Skevi e Volkov (2012).

O modelo possui a seguinte dinâmica. Considere um modelo a tempo discreto que tem como estado inicial o conjunto vazio. Em cada instante de tempo $n, n \in \mathbb{N}$, com probabilidade $p$ ocorre o nascimento de uma nova espécie e, com probabilidade $1-p$, há a morte de uma espécie (desde que o sistema não esteja vazio). Defina $X_{n}$ como sendo o número de espécies presentes no instante de tempo $n$. Observe que $X_{n}$ é um passeio aleatório em $\mathbb{Z}^{+}$que salta para a direita com probabilidade $p$ e para a esquerda com probabilidade $1-p$. Quando $X_{n}$ é igual a zero, o processo vai para o estado 1 com probabilidade $p$ ou permanece em 0 com probabilidade $1-p$. Para cada nova espécie que surge no sistema é associado a ela um valor aleatório, sorteado a partir de uma distribuição uniforme em [0,1]. Tais números aleatórios são considerados como sendo a aptidão (ou seja, o fitness) de cada espécie, que são independentes entre si. Em cada instante onde há um evento de morte, a espécie a ser 
extinta do sistema será aquela que possuir o fitness com valor mais baixo.

Em Guiol et al. (2011) é explorada a dinâmica do modelo GMS no sentido da distribuição dos fitness presentes no sistema após um longo período de tempo, quando $p>1 / 2$. Ou seja, quando $X_{n}$ é um passeio aleatório transiente (veja o apêndice A). Nesse contexto, foi mostrado pelos autores a existência de uma transição de fase e o conjunto das espécies com fitness acima do valor crítico se aproxima de uma distribuição uniforme, enquanto que todas as espécies com fitness abaixo do valor crítico desaparecem após um tempo finito aleatório.

\subsection{O modelo GMS com competição entre espécies}

Tendo em mente o modelo GMS apresentado na seção anterior, nosso objetivo será estudar a dinâmica do modelo quando $p<1 / 2$. Observe que, neste caso, o processo $X_{n}$ é recorrente e o sistema se torna vazio infinitas vezes. Uma questão interessante a se estudar é a distribuição do fitness do indivíduo mais bem adaptado (ou mais forte) em cada excursão antes do sistema se tornar vazio.

Deduzimos, explicitamente, a distribuição do fitness do indivíduo mais forte em uma excursão, ou seja, entre dois instante em que o sistema se torna vazio. A última espécie a ser extinta do sistema será a mais forte daquela excursão, uma vez que os indivíduos que já morreram possuiam uma aptidão menor para sobreviver ao meio.

Observe que excursões com tamanho 2 podem ocorrer e, neste caso, o único indivíduo que nasceu morre sem entrar em competição com nenhuma outra espécie. A fim de garantir que ao longo de uma excursão haja competição entre espécies, no sentido de ocorrer um processo de seleção natural, propomos a seguinte mudança para o modelo: Em cada instante de tempo após o sistema se tornar vazio, $m$ novas espécies independentes são introduzidas no sistema (ao invés de somente 1) com probabilidade $p$, ou o sistema permanece vazio com probabilidade $1-p$. Denotaremos tal variação do modelo por GMS(m). Assim, seguindo tal notação, GMS(1) é o modelo original.
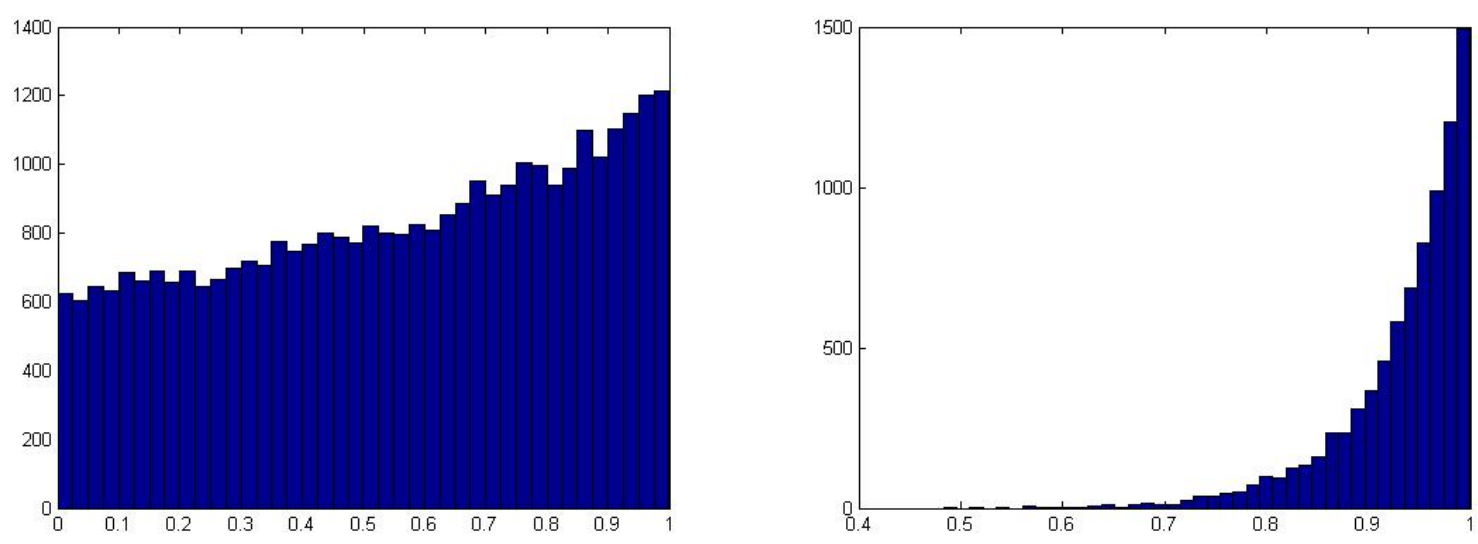

Figura 2.1: Histograma do fitness da espécie mais forte no GMS(1) e GMS(10), respectivamente, após 200.000 nascimentos e mortes para $p=1 / 4$. 
As figuras acima mostram o papel da competição na distribuição do fitness da espécie mais bem adaptada. Observe que excursões pequenas são mais comuns no GMS(1) do que no GMS(10). Assim, a competição introduzida em GMS(10) evita que indivíduos com fitness baixo sejam os mais fortes ao longo de uma excursão.

O resultado que segue apresenta a distribuição do fitness do último indivíduo antes do sistema se tornar vazio no modelo GMS(m). O resultado é apresentado em termos da função hipergeométrica de Gauss, que definiremos na sequência, maiores detalhes sobre tais funções podem ser encontradas em Luke (1969). A função hipergeométrica de Gauss é denotada por ${ }_{2} F_{1}(a, b ; c ; z)$, onde,

$$
{ }_{2} F_{1}(a, b ; c ; z)=\sum_{k \geq 0} \frac{(a)_{k}(b)_{k}}{(c)_{k}} \frac{z^{k}}{k !}, \quad|z|<1,
$$

$a, b, c$, são números reais com $c \notin \mathbb{Z}_{-}$, e $(a)_{k}$ é o coeficiente de Pochhammer, denotado por,

$$
(a)_{k}=a(a+1) \cdots(a+k-1), \quad(a)_{0}=1 .
$$

Teorema 2.2.1. Seja $p \leq 1 / 2, m \geq 1$ e $Z_{m}$ o fitness do último indivíduo no sistema antes dele se tornar vazio no modelo $G M S(m)$. Então $Z_{m}$ é uma variável aleatória com distribuição

$$
\mathbb{P}\left(Z_{m} \leq t\right)=(q t)^{m} F_{1}\left(\frac{m}{2}, \frac{m+1}{2} ; m+1 ; 4 p q t\right) \quad 0 \leq t<1 .
$$

Prova. Seja $\tau$ o comprimento de uma excursão do 0 para o 0 . Como $p<1 / 2$ temos que $\mathbb{P}(\tau<\infty)=1 \mathrm{e}$

$$
\mathbb{P}[\tau=k+1]=\mathbb{P}\left[T_{-m}=k\right]=\frac{m}{k}\left(\begin{array}{c}
k \\
\frac{k-m}{2}
\end{array}\right) p^{(k-m) / 2} q^{(k+m) / 2}, k \geq m, k+m \text { par },
$$

onde $T_{-m}$ é o tempo da primeira visita a $-\mathrm{m}$ no passeio aleatório em $\mathbb{Z}$ que tem seu início em 0 (tal resultado pode ser encontrado em Bhattacharya e Waymire (2009)).

Se $\tau=k+1$, então ocorrem $m+\frac{k-m}{2}$ nascimentos e $\frac{k+m}{2}$ mortes durante a excursão, onde a última corresponde a do indivíduo que possui o maior fitness dentre os $\frac{k+m}{2}$ nascidos. Logo,

$$
\mathbb{P}\left[Z_{m} \leq t\right]=\sum_{k=m}^{\infty} \mathbb{P}[\tau=k+1] \mathbb{P}\left[\max \left(Y_{1}, \ldots, Y_{\frac{m+k}{2}}\right) \leq t\right],
$$

onde $Y_{1}, \ldots, Y_{\frac{m+k}{2}}$ são v.a. i.i.d. uniformes em $[0,1]$. Portanto, 


$$
\begin{aligned}
\mathbb{P}\left[Z_{m} \leq t\right] & \left.=\sum_{k=m}^{\infty} \frac{m}{k}\left(\begin{array}{c}
k \\
\frac{k-m}{2}
\end{array}\right) p^{(k-m) / 2} q^{(k+m) / 2} t^{(k+m) / 2} \mathbf{1}_{\{m+k} \text { par }\right\} \\
& =\sum_{l=m}^{\infty} \frac{m}{2 l-m}\left(\begin{array}{c}
2 l-m \\
l
\end{array}\right) p^{l-m} q^{l} t^{l} \quad(k+m=2 l, l \geq m) \\
& =\sum_{j=0}^{\infty} \frac{m}{m+2 j}\left(\begin{array}{c}
m+2 j \\
m+j
\end{array}\right) p^{j} q^{m+j} t^{m+j} \quad(l=m+j) \\
& =(q t)^{m} \sum_{j=0}^{\infty} \frac{m+2 j}{m+\left(\begin{array}{c}
m+2 j \\
m+j
\end{array}\right)(p q t)^{j}} \\
& =(q t)^{m} \sum_{j=0}^{\infty} \frac{m}{m+2 j} \frac{(m+2 j) !}{(m+j) ! j !}(p q t)^{j} \\
& =(q t)^{m} \sum_{j=0}^{\infty} m \frac{(m+2 j-1) !}{(m+j) !} \frac{(p q t)^{j}}{j !} \\
& =(q t)^{m} \sum_{j=0}^{\infty} \frac{\left(\frac{m}{2}\right)_{j}\left(\frac{m+1}{2}\right)_{j} 2^{2 j} m(m-1) !}{(m+1)_{j} m !} \frac{(p q t)^{j}}{j !} \\
& =(q t)^{m} \sum_{j=0}^{\infty} \frac{\left(\frac{m}{2}\right)_{j}\left(\frac{m+1}{2}\right)_{j}}{(m+1)_{j}} \frac{(4 p q t)^{j}}{j !} \\
& \left(q F_{1}\left(\frac{m}{2}, \frac{m+1}{2} ; m+1 ; 4 p q t\right) .\right.
\end{aligned}
$$

Corolário 2.2.1. Seja $p<1 / 2$ e $Z$ o fitness do indivíduo mais forte antes do sistema se tornar vazio no modelo GMS(1). Então

$$
\mathbb{P}[Z \leq t]=\frac{1-\sqrt{1-4 p q t}}{2 p}, 0 \leq t<1 .
$$

Quando $p=1 / 2$, Z segue a distribuição $B(1,1 / 2)$.

Prova. Pelo Teorema 2.2.1 e tomando $m=1$ temos

$$
\begin{aligned}
\mathbb{P}[Z \leq t] & =(q t)_{2} F_{1}\left(\frac{1}{2}, 1 ; 2 ; 4 p q t\right) \\
& =\frac{1}{p} \sum_{j=0}^{\infty} \frac{\left(\frac{1}{2}\right)_{j}(1)_{j}}{(2)_{j}} \frac{4^{j}(p q t)^{j+1}}{j !} \\
& =\frac{1}{p} \sum_{j=0}^{\infty} \frac{(2 j) !}{(j+1) ! j !}(p q t)^{j+1} \\
& =\frac{1-\sqrt{1-4 p q t}}{2 p} .
\end{aligned}
$$


onde a equação (2.2) foi obtida usando o fato que $\left(\frac{1}{2}\right)_{j}(1)_{j}=2^{-2 j}(2 j)$ ! e a (2.3) usando o resultado contido em Prudnikov et al. (2002), Eq. 5.2.13.9 .

Por fim, temos o corolário abaixo referente ao valor esperado da variável aleatória $Z_{m}$.

Corolário 2.2.2. $\mathbb{E}\left[Z_{m}\right]=1-\frac{q^{m}}{m+1}{ }_{2} F_{1}\left(\frac{m}{2}, \frac{m+1}{2} ; m+1 ; 4 p q\right)$.

Prova.

$$
\begin{aligned}
\mathbb{E}\left[Z_{m}\right] & =\int_{0}^{1} \mathbb{P}\left[Z_{m}>t\right] d t \\
& =1-q^{m} \int_{0}^{1} t^{m}{ }_{2} F_{1}\left(\frac{m}{2}, \frac{m+1}{2} ; m+1 ; 4 p q t\right) d t \\
& =1-\frac{q^{m}}{m+1}{ }_{2} F_{1}\left(\frac{m}{2}, \frac{m+1}{2} ; m+2 ; 4 p q\right)
\end{aligned}
$$

onde a última linha foi obtida usando o resultado dado em Gradshteyn e Ryzhik (2007) [Eq. 7.512.11].

Observação 2.2.1. Quando $p=1 / 2$, usando a equação (veja [Eq. 7.512.11], Gradshteyn e Ryzhik (2007))

$$
{ }_{2} F_{1}(\alpha, \beta ; \gamma ; 1)=\frac{\Gamma(\gamma) \Gamma(\gamma-\alpha-\beta)}{\Gamma(\gamma-\alpha) \Gamma(\gamma-\beta)}
$$

temos que

$$
\begin{aligned}
\mathbb{E}\left[Z_{m}\right] & =1-\frac{q^{m}}{m+1}{ }_{2} F_{1}\left(\frac{m}{2}, \frac{m+1}{2} ; m+2 ; 1\right) \\
& =1-\frac{1}{2^{m}(m+1)}\left[\frac{\Gamma(m+2) \Gamma(3 / 2)}{\Gamma\left(\frac{m+3}{2}+\frac{1}{2}\right) \Gamma\left(\frac{m+3}{2}\right)}\right] \\
& =1-\frac{\sqrt{\pi} m !}{2^{m+1} \Gamma\left(\frac{m+3}{2}+\frac{1}{2}\right) \Gamma\left(\frac{m+3}{2}\right)}
\end{aligned}
$$

onde a última linha foi obtida usando $\Gamma(3 / 2)=\sqrt{\pi} / 2$. Agora, usando

$$
\Gamma(2 z)=\frac{\Gamma\left(z+\frac{1}{2}\right) \Gamma(z)}{2^{1-2 z} \sqrt{\pi}}
$$

temos

$$
\mathbb{E}\left[Z_{m}\right]=1-\frac{2}{(m+1)(m+2)}
$$


8 O MODELO GUIOL-MACHADO-SCHINAZI COM DINÂMICA DE COMPETIÇÃO ENTRE ESPÉCIES 


\section{Capítulo 3}

\section{Processo estocástico para evolução por meio de mutação}

Neste capítulo apresentamos uma nova proposta de modelo estocástico para a evolução de espécies, cujo processo evolutivo é dado por meio de mutação. O modelo proposto segue as ideias apresentadas por Guiol et al. (2011) e por Schinazi e Schweinsberg (2008). Podemos pensar nesse modelo como a sobrevivência de um patógeno no organismo, cuja estratégia usada para tentar sobreviver é a mutação. O grafo sobre o qual o modelo evolui é a árvore binária orientada com raiz. Vamos considerar duas dinâmicas diferentes para a evolução do processo infeccioso, uma determinística e a outra aleatória. O modelo é dito determinístico no sentido de que o preenchimento dos ramos pelas partículas ocorre sempre de um mesmo modo. Neste contexto, dizer que o modelo sobre a árvore é aleatório significa que a ocupação dos vértices pelas partículas passa a ser aleatória.

Tanto para o caso determinístico, quanto para o caso aleatório analisamos a transição de fase do modelo primeiramente sobre um ramo fixado e, em um segundo momento para a árvore como um todo. Em ambos os casos provamos a existência de transição de fase no modelo.

Uma motivação para propormos tal modelo vem da infecção causada por patógenos, que são organismos capazes de gerar doenças infecciosas aos seus hospedeiros. Como exemplos de agentes infecciosos temos os vírus e as bactérias. Pelo apresentado no Capítulo 1 , possuímos um complexo sistema responsável pela defesa de nosso organismo quando há a presença de agentes estranhos, como os patógenos. Tal sistema, denominado sistema imunológico, tem a capacidade de memorizar aqueles patógenos que já nos infectaram. Assim, uma estratégia muito utilizada, por exemplo, pelos vírus, para tentar sobreviver é a evolução por meio de mutação. Quando um vírus sofre mutação, o sistema imunológico gasta um determinado tempo para conseguir produzir anticorpos capazes de eliminar essa nova partícula. O mesmo ocorre com as bactérias, que ao mutar, podem se tornar cada vez mais fortes contra a ação do sistema imunológico, fazendo com que o sistema precise, novamente, criar anticorpos mais especializados, a fim de combater a infecção. 
O modelo evolui a tempo contínuo sobre a árvore binária e, no instante inicial $t=0$ o processo começa com um único patógeno na raiz, que rotularemos como sendo do tipo 1. Associamos a raiz da árvore um Processo Pontual de Poisson de taxa constante $\lambda>0$ $(\operatorname{PPP}(\lambda))$ e, a cada marca desse processo um novo patógeno é incorporado ao sistema. Consideramos, também, que cada novo patógeno poderá ser igual a seu progenitor, ou seja, do mesmo tipo, ou uma mutação. Em instantes de nascimento, com probabilidade $1-r$ é gerado um patógeno de mesmo tipo que seu progenitor, que se acumula na raiz e com probabilidade $r$ o patógeno será de um tipo acima e ocupará um dos sítios imediatamente abaixo da raiz. De modo geral, se um patógeno é do tipo $i$, seus descendentes serão do tipo $i$ com probabilidade $1-r$ e ocuparão o mesmo vértice que o patógeno progenitor. Mas, se for gerado um patógeno do tipo $i+1$, com probabilidade $r$, será uma mutação e ocupará um dos vértices imediatamente abaixo do seu progenitor. Perceba que nos preocupamos apenas com as duas primeiras marcas do $\operatorname{PPP}(\lambda)$ que geram mutações, uma vez que nosso objetivo é o preenchimento do grafo.

Quando um vértice que estava vazio é ocupado por um novo tipo, associamos um novo $\operatorname{PPP}(\lambda)$ a aquele sítio, independente de tudo e, desse modo, a linhagem desse patógeno se desenvolverá de modo independente das demais presentes no sistema.

Para os instantes de morte do processo, que representam a ação do sistema imunológico no combate ao patógeno presente no organismo, consideramos um Processo Pontual de Poisson de taxa $1(\mathrm{PPP}(1))$, que é independente dos demais processos associados ao sistema. Na ocorrência de uma marca deste processo a espécie de menor fitness, viva naquele instante de tempo, será eliminada em sua totalidade. Ou seja, aquele fitness passa a não pertencer mais ao sistema.

Denotando por $X(t)$ o número de tipos presentes no sistema no instante $t$. O processo (ou a infecção) estará extinto no instante $t_{0}>0$ quando não há nenhum patógeno vivo no sistema, ou seja, $X\left(t_{0}\right)=0$. Observe que $t_{0}$ é o primeiro instante em que o sistema se torna vazio, pois o estado 0 é absorvente para o processo.

Se $X(t)>0$ para todo instante de tempo $t \geq 0$, então diremos que o processo sobrevive.

\subsection{Processo de infecção para o caso determinístico}

Considerando o modelo apresentado acima. No instante de tempo $t=0$, a raiz é ocupada por um único patógeno do tipo 1. O patógeno espera um tempo exponencial de taxa $\lambda$ para gerar novos indivíduos. Em instantes de nascimento, com probabilidade $1-r$ ele gera um patógeno de mesmo tipo que se acumula na raiz e com probabilidade $r$ o patógeno será do tipo 2 e ocupará o sítio abaixo da raiz, à esquerda. No próximo instante de nascimento do indivíduo do tipo 1, com probabilidade $r$ o patógeno será novamente do tipo 2, no entanto, ele ocupará o vértice do ramo à direita. De modo geral, se um patógeno é do tipo $i$, seus descendentes serão do tipo $i$ com probabilidade $1-r$ e ocuparão o mesmo vértice que o 
patógeno progenitor. Mas, se for gerado um patógeno do tipo $i+1$, com probabilidade $r$, então o primeiro a surgir ocupará o vértice imediatamente abaixo do seu progenitor à esquerda e, o segundo patógeno gerado do tipo $i+1$ ocupará o vértice imediatamente abaixo do seu progenitor à direita.

Como apresentado acima, os instantes de morte ocorrem de acordo com um processo pontual de Poisson de taxa 1, que evolui paralelamente aos processos de nascimento e é único para todos os patógenos. Em uma marca do processo de morte, todos os patógenos do mesmo tipo serão totalmente eliminados do processo.

\subsubsection{Análise de sobrevivência considerando um ramo fixo}

Ao fixarmos um caminho ao longo da árvore queremos estudar a sobrevivência do processo de infecção ao longo desse ramo. Desse modo, obtemos o seguinte resultado.

Teorema 3.1.1. Seja $\lambda>0,0<r<1$ e fixe $r$. Para qualquer ramo fixo escolhido na árvore, tendo como início a raiz, temos que:

a) Se $\lambda \leq 1 / r$ o processo de infecção é extinto com probabilidade 1 .

b) Se $\lambda>\frac{1+\sqrt{2}}{r}$ o processo sobrevive com probabilidade positiva.

Prova. Escolha, primeiramente o ramo extremo à esquerda, isto é, só andamos para a esquerda na árvore. Seja $X(t)$ o número de diferentes tipos de patógenos vivos no tempo $t \geq 0$ neste ramo. Defina

$$
T_{0}:=\inf \{t: X(t)=2\}
$$

isto é, o instante de tempo no qual surge o segundo tipo no sistema. Com probabilidade positiva o primeiro patógeno (presente no instante $t=0$ ) gera a um novo tipo de patógeno antes de morrer.

De fato, seja $t_{M_{1}}$ o primeiro instante de morte, isto é, o instante no qual o primeiro patógeno presente no sistema é eliminando. Pela dinâmica do modelo, este instante é a primeira marca de um processo de Poisson de taxa $\lambda r$. Assim, temos que a probabilidade do evento $\left\{T_{0}<t_{M_{1}}\right\}$ é igual a $\frac{\lambda r}{1+\lambda r}$. Pelo afirmado acima, podemos concluir que $\mathbb{P}\left(T_{0}<\infty\right)>0$.

Defina, por indução, para cada inteiro positivo, no evento em que $T_{n-1}$ é finito, o tempo de parada

$$
T_{n}=\inf \left\{t>T_{n-1}: X(t) \neq X\left(T_{n-1}\right)\right\} .
$$

Isto é, $T_{n}$ é o primeiro instante após $T_{n-1}$ no qual ou surge um novo patógeno, neste caso $X\left(T_{n}\right)=X\left(T_{n-1}\right)+1$, ou o tipo do patógeno mais antigo é eliminado e, então, $X\left(T_{n}\right)=$ $X\left(T_{n-1}\right)-1$. Observe que, independentemente da quantidade de patógenos no sistema, desde que $X(t)>0$, novos tipos surgem à taxa $\lambda r$ e o tipo do patógeno mais antigo é eliminado 
por completo a taxa 1. Seja $\hat{r}=\frac{\lambda r}{1+\lambda r}$, temos que $\mathbb{P}\left[X\left(T_{n}\right)=k+1 \mid X\left(T_{n-1}\right)=k\right]=\hat{r}$. Agora, considere a cadeia de nascimento e morte $\left(Y_{n}\right)_{n \geq 0}$ tal que $Y_{0}=2$ e $\forall k \geq 0$

$$
\left.\left.\mathbb{P}\left[Y_{n}=k+1 \mid Y_{n-1}\right)=k\right]=\hat{r} \quad \text { e } \quad \mathbb{P}\left(Y_{n}=k-1 \mid Y_{n-1}\right)=k\right)=1-\hat{r}
$$

Temos que as cadeias $\left(X\left(T_{n}\right)\right)_{n \geq 0}$ e $\left(Y_{n}\right)_{n \geq 0}$ possuem a mesma quantidade de indivíduos até o instante em que atingem o zero, pois, nesse momento, a cadeia $\left(X\left(T_{n}\right)\right)$ permanecerá para sempre no estado 0 com probabilidade 1 , enquanto que a cadeia $\left(Y_{n}\right)$ eventualmente sairá do estado 0 com probabilidade $\hat{r}$.

Para provarmos a existência do valor crítico, basta mostrar que a cadeia $\left(Y_{n}\right)_{n \geq 0}$ é recorrente se $\lambda r \leq 1$, nos garantindo que eventualmente ela atingirá o zero, o que implica a não sobrevivência do processo de infecção para a cadeia $\left(X\left(T_{n}\right)\right)$.

Para provar a recorrência, considere a Proposição III 4.1 de Schinazi (1999) (ver C.3). Assim, o processo será recorrente se, e somente se,

$$
\sum_{i=1}^{\infty}\left(\frac{1-\hat{r}}{\hat{r}}\right)^{i}=\sum_{i=1}^{\infty}\left(\frac{1}{\lambda r}\right)^{i}=+\infty .
$$

Ou seja, se $\lambda r \leq 1$ o processo será recorrente e, como consequência, com probabilidade 1 o processo $\left(X\left(T_{n}\right)\right)$ atingirá o estado zero. Caso contrário o processo será transiente e, com probabilidade positiva a infecção sobreviverá.

Considere, agora, o ramo extremo à direita e tome $X(t)$ como sendo o número de patógenos nesse ramo. Observe que o tempo entre o surgimento do tipo i e do tipo i+1 nesse ramo é aleatório e tem distribuição $\operatorname{Gama}(2, \lambda r)$, enquanto que os tempos entre dois eventos de morte tem distribuição exponencial de parâmetro 1 . Assim, sendo $G_{2, \lambda r}$ a variável aleatória gama de parâmetros 2 e $\lambda r$ e $E_{1}$ a variável exponencial temos que

$$
\mathbb{P}(X(t+s)=k+1 \mid X(t)=k)=\mathbb{P}\left(G_{2, \lambda p}<E_{1}\right)=\left(\frac{\lambda p}{1+\lambda r}\right)^{2}
$$

e

$$
\mathbb{P}(X(t+s)=k-1 \mid X(t)=k)=\mathbb{P}\left(E_{1}<G_{2, \lambda p}\right)=1-\left(\frac{\lambda p}{1+\lambda r}\right)^{2} .
$$

Podemos acoplar esse processo a uma cadeia de nascimento e morte de modo análogo como fizemos anteriormente. Assim, basta analisarmos a recorrência e a transiência da cadeia de nascimento e morte para obtermos o valor crítico do modelo em questão.

Então, considere a cadeia de modo que com probabilidade $\bar{p}_{i, i+1}=\left(\frac{\lambda r}{1+\lambda r}\right)^{2}$, se $i \geq 0$, há o surgimento de um novo tipo e com probabilidade $\bar{p}_{i, i-1}=1-\left(\frac{\lambda r}{1+\lambda r}\right)^{2}$, se $i \geq 1$ há a extinção de um tipo existente. Novamente pela Proposição III 4.1 de Schinazi (1999), segue que a 
cadeia de nascimento e morte é recorrente se, e somente se,

$$
\sum_{i=1}^{\infty}\left(\frac{1-\frac{(\lambda r)^{2}}{(1+\lambda r)^{2}}}{\frac{(\lambda r)^{2}}{(1+\lambda r)^{2}}}\right)^{i}=\infty
$$

Ou seja, se

$$
\frac{(1+\lambda r)^{2}-(\lambda r)^{2}}{(\lambda r)^{2}}>1
$$

Temos que, $\lambda r \leq 1+\sqrt{2}$.

Por fim, observe que para qualquer caminho que escolhemos, esperamos um tempo exponencial de taxa $\lambda r$ para que surja um novo patógeno no ramo abaixo, à esquerda e um tempo gama de taxas 2 e $\lambda r$ para que o novo patógeno vá para o ramo abaixo, à direita. Assim, sendo $\alpha_{i}$ a probabilidade de surgir um novo patógeno no próximo ramo do caminho, dado que há $i$ patógenos distintos ao longo do ramo já percorrido e $\beta_{i}=1-\alpha_{i}$, em símbolos, $\alpha_{i}=p_{i, i+1}$ e $\beta_{i}=p_{i, i-1}$. Sendo, ainda, $\alpha_{d}$ a probabilidade como descrita acima, mas agora considerando o passeio pelo ramo que só dá passos à direita e $\alpha_{e}$ somente com passos à esquerda, segue que

$$
\alpha_{d} \leq \alpha_{i} \leq \alpha_{e}
$$

$\mathrm{e}$

$$
\beta_{e} \leq \beta_{i} \leq \beta_{d}
$$

Logo,

$$
\sum_{i=1}^{\infty}\left(\frac{\beta_{e}}{\alpha_{e}}\right)^{i} \leq \sum_{i=1}^{\infty} \prod_{k=1}^{i} \frac{\beta_{i}}{\alpha_{i}} \leq \sum_{i=1}^{\infty}\left(\frac{\beta_{d}}{\alpha_{d}}\right)^{i}
$$

Pelo apresentado acima, se $\lambda r>1+\sqrt{2}$ então $\sum_{i=1}^{\infty}\left(\frac{\beta_{d}}{\alpha_{d}}\right)^{i}<\infty$. Portanto, $\sum_{i=1}^{\infty} \prod_{k=1}^{i} \frac{\beta_{i}}{\alpha_{i}}<\infty$, fazendo com que a cadeia seja transiente. Como consequência, o processo de infecção também será transiente e com probabilidade positiva ele sobrevive.

Além disso, temos que se $\lambda r \leq 1$ segue que $\sum_{i=1}^{\infty}\left(\frac{\beta_{e}}{\alpha_{e}}\right)^{i}=\infty$. Logo, $\sum_{i=1}^{\infty} \prod_{k=1}^{i} \frac{\beta_{i}}{\alpha_{i}}=\infty$. Mostrando que a cadeia é recorrente nesse caso. Por argumentos análogos aos apresentados anteriormente, segue que com probabilidade 1 o processo de infecção será extinto nessas condições.

O gráfico abaixo apresenta as curvas $\lambda=\frac{1}{r}$ e $\lambda=\frac{1+\sqrt{2}}{r}$. Pelo apresentado no teorema, para qualquer processo com taxa na região A, com probabilidade 1 ele será extinto em todos os ramos e, com taxas na região C, ele terá probabilidade positiva de sobrevivência em todos os ramos.

Observe que, pelo apresentado acima, uma vez que fixamos o caminho, conseguimos 


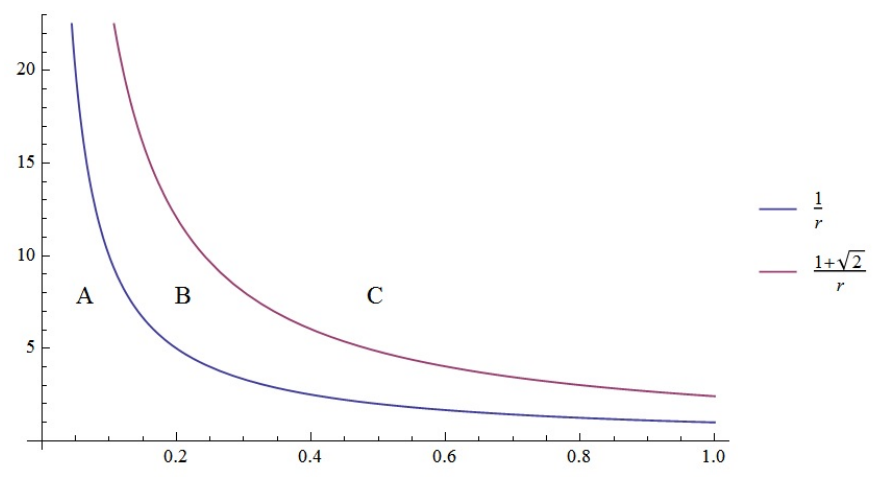

Figura 3.1: Gráfico das funções $\lambda=\frac{1}{r}$ e $\lambda=\frac{1+\sqrt{2}}{r}$, respectivamente.

provar a existência de um parâmetro crítico $\lambda_{c}$. Como ilustrado no exemplo abaixo:

Exemplo 3.1.1. Considere o ramo formado pelo seguinte caminho ao longo da árvore. Partindo da raiz o primeiro passo é para a esquerda, o segundo para a direita, o terceiro à esquerda, o quarto à direita e assim por diante. Para este ramo temos $\lambda_{c}=\frac{1+\sqrt{5}}{2 r}$.

De fato, observe que para o ramo ser ocupado por espécies nos vértices à esquerda, sendo a raiz o vértice 0, o tempo de espera é sempre exponencial de taxa $\lambda r$, enquanto que para um vértice á direita ser ocupado, o tempo de espera será uma variável aleatória Gama de taxas 2 e $\lambda r$. Defina $\alpha_{1}$ e $\beta_{1}$ as probabilidade de nascimento e morte, respectivamente, quando consideramos que os instantes entre dois nascimentos ocorrem de acordo com uma variável aleatória exponencial de taxa $\lambda r$, ou seja,

$$
\left\{\begin{array}{l}
\alpha_{1}=p_{i, i+1}=\frac{\lambda r}{1+\lambda r} \\
\beta_{1}=p_{i, i-1}=\frac{1}{1+\lambda r}
\end{array}\right.
$$

para $i$ ímpar.

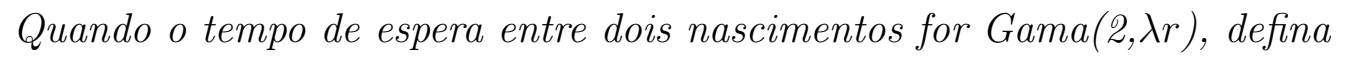

$$
\left\{\begin{array}{l}
\alpha_{2}=p_{i, i+1}=\left(\frac{\lambda r}{1+\lambda r}\right)^{2} \\
\beta_{2}=p_{i, i-1}=1-\left(\frac{\lambda}{1+\lambda r}\right)^{2}
\end{array},\right.
$$

para $i$ par.

Assim, usando as relações apresentadas na prova do Teorema 3.1.1, temos que nessas condições o processo é recorrente se, e somente se,

$$
\sum_{i=1}^{+\infty} P_{i}=+\infty
$$

onde $P_{i}=\prod_{j=1}^{i} \frac{\beta_{i}}{\alpha_{i}}$. 
Logo,

$$
\begin{aligned}
\sum_{i=1}^{+\infty} P_{i} & =\sum_{i=1}^{+\infty}\left[\left(\frac{\beta_{1}}{\alpha_{1}}\right)^{i}\left(\frac{\beta_{2}}{\alpha_{2}}\right)^{i-1}+\left(\frac{\beta_{1}}{\alpha_{1}}\right)^{i}\left(\frac{\beta_{2}}{\alpha_{2}}\right)^{i}\right] \\
& =\frac{\beta_{1}}{\alpha_{1}}+\left(\frac{\beta_{1}}{\alpha_{1}}+1\right) \sum_{i=1}^{+\infty}\left(\frac{\beta_{1} \beta_{2}}{\alpha_{1} \alpha_{2}}\right)^{i}=+\infty \Leftrightarrow \frac{\beta_{1} \beta_{2}}{\alpha_{1} \alpha_{2}} \geq 1
\end{aligned}
$$

Assim,

$$
\begin{aligned}
\frac{\beta_{1} \beta_{2}}{\alpha_{1} \alpha_{2}} \geq 1 & \Leftrightarrow \frac{\frac{1}{1+\lambda r}}{\frac{\lambda r}{1+\lambda r}} \cdot \frac{1-\left(\frac{\lambda r}{1+\lambda r}\right)^{2}}{\left(\frac{\lambda r}{1+\lambda r}\right)^{2}} \geq 1 \\
& \Leftrightarrow(\lambda r)^{3}-2 \lambda r-1 \leq 0 \\
& \Leftrightarrow \lambda r \leq \frac{1+\sqrt{5}}{2}
\end{aligned}
$$

Portanto, se $0<\lambda r \leq \frac{1+\sqrt{5}}{2}$, o processo de infecção nesse ramo morrerá com probabilidade e se $\lambda r>\frac{1+\sqrt{5}}{2}$ há sobrevivência com probabilidade positiva.

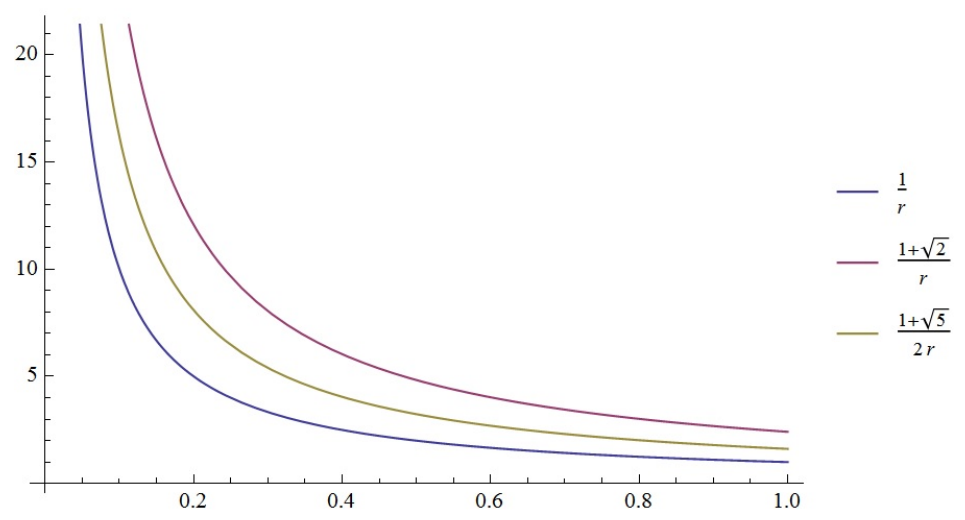

Figura 3.2: Gráfico das funções $\lambda=\frac{1}{r}, \lambda=\frac{1+\sqrt{5}}{2 r}$ e $\lambda=\frac{1+\sqrt{2}}{r}$, respectivamente.

Para o caminho escolhido acima, cada passo foi estabelecido deterministicamente. O próximo teorema mostrar que, se fixarmos um caminho aleatório, então também conseguimos um $\lambda_{c}$. No entanto, o parâmetro crítico depende da aleatoriedade do caminho.

Teorema 3.1.2. Seja $\left(A_{n}\right)_{n \geq 1}$ um passeio aleatório simples sobre a árvore binária com raiz tal que com probabilidade $q$ a partícula se move para o ramo abaixo à esquerda e com probabilidade 1 - q para o ramo abaixo à direita. Fixando o ramo dado pela realização do passeio aleatório $A_{n}$ temos que existe um $\lambda$ crítico, $\lambda_{c}(q)=\frac{(1-q)+\sqrt{q^{2}-2 q+2}}{r}$ tal que se $\lambda \leq \lambda_{c}$ o processo de infecção sobre o ramo fixado morre com probabilidade 1 e, se $\lambda>\lambda_{c}$ há uma probabilidade positiva de sobrevivência da infecção no ramo.

Observação 3.1.1. Observe que quando $q=0$ o ramo fixado é aquele em que todos os passos são à direita e, pelos Teoremas 3.1.1 e 3.1.2 temos que $\lambda_{c}=\frac{1+\sqrt{2}}{r}$. Quando $q=1$, o 
ramo fixado é aquele em qual todos os passos são à esquerda, logo temos que $\lambda_{c}=\frac{1}{r}$, como já tínhamos provado nos Teoremas 3.1.1 e 3.1.2 .

Prova. Fixe um caminho aleatório e seja $i$ o número de partículas presentes nesse caminho em um instante de tempo $t \geq 0$. Observe que

$$
\begin{gathered}
i \longrightarrow i+1, \text { com probabilidade }\left(\frac{\lambda r}{1+\lambda r}\right)^{2}(1-q)+\frac{\lambda r}{1+\lambda r} q \\
i \longrightarrow i-1, \text { com probabilidade }\left(1-\frac{\lambda r}{1+\lambda r}\right)^{2}(1-q)+\frac{1}{1+\lambda r} q
\end{gathered}
$$

Essas probabilidades dependem do caminho que está sendo considerado, isto é, se foi originada uma partícula à direita ou à esquerda.

Temos que o processo descrito pelas probabilidades de transição acima é uma cadeia de nascimento e morte que tem 0 como estado absorvente. Assim, novamente pela Proposição III 4.1 de Schinazi (1999) temos que o processo será transiente se, e somente se,

$$
\begin{gathered}
\sum_{i=1}^{\infty} \prod_{k=1}^{i}\left(\frac{\left(1-\frac{\lambda r}{1+\lambda r}\right)^{2}(1-q)+\frac{1}{1+\lambda r} q}{\left(\frac{\lambda r}{1+\lambda r}\right)^{2}(1-q)+\frac{\lambda r}{1+\lambda r} q}<+\infty\right. \\
\Longleftrightarrow \frac{\left(1-\frac{\lambda r}{1+\lambda r}\right)^{2}(1-q)+\frac{1}{1+\lambda r} q}{\left(\frac{\lambda r}{1+\lambda r}\right)^{2}(1-q)+\frac{\lambda r}{1+\lambda r} q}<1 \\
\Longleftrightarrow(\lambda r)^{2}+(2 q-2) \lambda r-1>0 \\
\Longleftrightarrow \lambda r \leq 1-q+\sqrt{q^{2}-2 q+2}
\end{gathered}
$$

Logo, para cada $q$ e $r$ fixos, temos um parâmetro crítico $\lambda_{c}=\frac{1-q+\sqrt{q^{2}-2 q+2}}{r}$ de modo que, abaixo de $\lambda_{c}$ o processo é extinto com probabilidade 1 e para valores maiores que $\lambda_{c}$ há uma probabilidade positiva de sobrevivência.

Observação 3.1.2. Fixe, agora, o caminho extremo à esquerda. Queremos entender o comportamento do processo sobre esses ramos em relação à quantidade de indivíduos de cada tipo para um instante de tempo $t$ quando $\lambda r>1$. Definindo $X(t)$ como sendo o número de tipos sobre o ramo fixado, perceba que $X(t)$ é uma cadeia de nascimento e morte a tempo contínuo e as probabilidades de transição de estado são:

$$
\begin{gathered}
p_{i}=\mathbb{P}[X(t+h)=i+1 \mid X(t)=i]=\frac{\lambda r}{1+\lambda r}, i=1,2, \ldots \\
p_{0}=\mathbb{P}[X(t+h)=1 \mid X(t)=0]=1 \\
q_{i}=\mathbb{P}[X(t+h)=i-1 \mid X(t)=i]=\frac{1}{1+\lambda r}, i=1,2, \ldots
\end{gathered}
$$




$$
q_{0}=\mathbb{P}[X(t+h)=-1 \mid X(t)=0]=0 .
$$

Assim, se $\lambda r>1$ temos que o processo $\{X(t)\}$ é transiente.

Para estudarmos a quantidade de indivíduos em cada tipo considere o vetor aleatório infinito

$$
\tilde{X}(t):=\left(X_{1}(t), X_{2}(t), \ldots\right)
$$

onde $X_{i}(t)$ representa o número de indivíduos do tipo $i$ no instante $t$, com $i=\{1,2, \ldots\}$.

Queremos determinar o valor médio do número de indivíduos para cada um dos tipos em um determinado instante de tempo $t$.

Temos 3 casos a considerar:

Caso 1) $k=\min \left\{i: X_{i}(t)>0\right\}$

Caso 2) $\min \left\{i: X_{i}(t)>0\right\}<k \leq \max \left\{i: X_{i}(t)>0\right\}$

Caso 3) $k-1=\max \left\{i: X_{i}(t)>0\right\}$

onde $k$ representa o tipo para cada um dos casos acima.

\section{Caso 1}

Fixe um tempo $t>0$ e considere o tipo $k$ tal que $k=\min \left\{i: X_{i}(t)>0\right\}$, isto é, o tipo mais a esquerda vivo no instante de tempo $t$, temos que

$$
\mathbb{E}\left[X_{k}(t)\right]=\lambda(1-r)-[\lambda(1-r)-1] e^{-t}
$$

Pois,

$$
X_{k}(t+h)-X_{k}(t) \mid \tilde{X}(t)=\left\{\begin{array}{rll}
1, & \text { com taxa } & \lambda(1-r) h+o(h) \\
-X_{k}(t), & \text { com taxa } & h+o(h) \\
0, & \text { com taxa } & 1-\lambda(1-r) h-h+o(h)
\end{array}\right.
$$

onde

$$
\lim _{h \rightarrow 0} \frac{o(h)}{h}=0
$$

Assim,

$$
\mathbb{E}\left[X_{k}(t+h)-X_{k}(t) \mid \tilde{X}(t)\right]=\lambda(1-r) h-h X_{k}(t)+o(h) .
$$

Donde segue que

$$
\mathbb{E}\left[X_{k}(t+h)-X_{k}(t)\right]=\lambda(1-r) h-h \mathbb{E}\left[X_{k}(t)\right]+o(h) .
$$


Defina $\tilde{x}_{k}(t):=\mathbb{E}\left[X_{k}(t)\right]$. Por (3.2) temos

$$
\begin{aligned}
\frac{d \tilde{x}_{k}(t)}{d t} & =\lim _{h \rightarrow 0} \frac{\mathbb{E}\left[X_{k}(t+h)-X_{k}(t)\right]}{h} \\
& =\lim _{h \rightarrow 0} \frac{\lambda(1-r) h-h \tilde{x}_{k}(t)+o(h)}{h} \\
& =\lambda(1-r)-\tilde{x}_{k}(t) .
\end{aligned}
$$

Relembre que estamos no caso quando $k$ representa o tipo mais a esquerda presente no instante $t$, deste modo, pela definição do modelo temos que $\tilde{x}_{k}(0)=1$. Logo, $\mathbb{E}\left[X_{k}(t)\right]$ será a solução do seguinte problema de valor inicial (PVI)

$$
\left\{\begin{array}{lll}
\tilde{x}_{k}^{\prime}(t) & = & \lambda(1-r)-\tilde{x}_{k}(t) \\
\tilde{x}_{k}(0) & = & 1
\end{array}\right.
$$

Nosso próximo passo é encontrar a solução do PVI acima. Então,

$$
\begin{aligned}
\frac{d \tilde{x}_{k}}{d t}=\lambda(1-r)-\tilde{x}_{k} & \Leftrightarrow \frac{d \tilde{x}_{k}}{\lambda(1-r)-\tilde{x}_{k}}=d t \\
& \Leftrightarrow \int \frac{d \tilde{x}_{k}}{\lambda(1-r)-\tilde{x}_{k}}=\int d t \\
& \Leftrightarrow-\ln \left(\lambda(1-r)-\tilde{x}_{k}\right)=t+C,
\end{aligned}
$$

onde C é uma constante. Aplicando a função exponencial em ambos os lados da igualdade em (3.4) temos que

$$
\tilde{x}_{k}(t)=\lambda(1-r)-C_{1} e^{-t}
$$

Usando o fato que $\tilde{x}_{k}(t)=1$, segue que a constante $C_{1}=-1+\lambda(1-r)$. Portanto,

$$
\tilde{x}_{k}(t)=\lambda(1-r)-[\lambda(1-r)-1] e^{-t}
$$

\section{Caso 2}

Fixe agora $k$ de modo que

$$
\min \left\{i: X_{i}(t)>0\right\}<k \leq \max \left\{i: X_{i}(t)>0\right\}
$$

isto é, $k$ representa todos os tipos existentes com exceção do mais a esquerda. Temos

$$
\mathbb{E}\left[X_{k}(t)\right]=\lambda t
$$

De fato,

$$
X_{k}(t+h)-X_{k}(t) \mid \tilde{X}(t)=\left\{\begin{array}{lll}
1, & \text { com taxa } & \lambda h+o(h) \\
0, & \text { com taxa } & 1-\lambda h+o(h)
\end{array}\right.
$$


Procedendo de modo análogo ao caso anterior e lembrando, da definição do modelo que nestas condiçôes $\tilde{x}_{k}(0)=0$, obtemos o PVI

$$
\left\{\begin{array}{l}
\tilde{x}_{k}^{\prime}(t)=\lambda \\
\tilde{x}_{k}(0)=0
\end{array}\right.
$$

Cuja solução será

$$
\tilde{x}_{k}(t)=\lambda t
$$

\section{Caso 3}

Este último caso representa o novo tipo que irá surgir no sistema.

Seja $k-1=\max \left\{i: X_{i}(t)>0\right\}$ temos,

$$
\mathbb{E}\left[X_{k}(t)\right]=\lambda r t
$$

A prova de tal afirmação segue idêntica a anterior a menos da seguinte alteração

$$
X_{k}(t+h)-X_{k}(t) \mid \tilde{X}(t)=\left\{\begin{array}{lll}
1, & \text { com taxa } & \lambda r h+o(h) \\
0, & \text { com taxa } & 1-\lambda r h+o(h)
\end{array} .\right.
$$

Definindo $\mathbb{E}[\tilde{X}(t)]=\left(\mathbb{E}\left[X_{1}(t)\right], \mathbb{E}\left[X_{2}(t)\right], \ldots\right)$, e pelos resultados anteriores segue que

$$
\mathbb{E}[\tilde{X}(t)]=\left(0, \cdots, 0, \lambda(1-r)-(\lambda(1-r)-1) e^{-t}, \lambda t, \cdots, \lambda t, 0, \cdots, 0\right) .
$$

\subsubsection{Análise de extinção e sobrevivência para o processo na árvore}

Acima fixamos um ramo e analisamos condições para a sobrevivência e extinção do modelo sobre ele. Observe que se o ramo fixado sobrevive, então garantimos a sobrevivência do modelo. No entanto, a não sobrevivência do ramo não garante a extinção do modelo. Assim, nosso próximo passo será determinar um parâmetro crítico de modo a garantir critérios para a extinção ou sobrevivência da infecção na árvore binária. Um modelo semelhante ao proposto aqui foi apresentado por Aldous e Krebs (1990), e a prova apresentada nessa seção segue as ideias propostas pelos autores.

Teorema 3.1.3. Seja $\lambda>0$ e $0<r<1$. Se $\lambda r \leq \min _{0<u<1}\left\{\frac{1}{1-u}\left[\frac{\lambda r}{\lambda r+u}+\left(\frac{\lambda r}{\lambda r+u}\right)^{2}\right]\right\}$, então o processo de evolução será extinto com probabilidade 1. Se $\lambda r>\min _{0<u<1}\left\{\frac{1}{1-u}\left[\frac{\lambda r}{\lambda r+u}+\left(\frac{\lambda r}{\lambda r+u}\right)^{2}\right]\right\}$, então o processo de evolução sobrevive com probabilidade positiva.

Prova.Vamos começar provando a extinção do processo. Seja $\left\{B_{\nu i}\right\}, \nu, i=1,2, \ldots$, variáveis aleatórias, independentes, com distribuição exponencial de parâmetro $\lambda r$ e $\left\{K_{i}\right\}, i=1,2, \ldots$, variáveis aleatórias, independentes, com distribuição exponencial de parâmetro 1. Seja, ainda, $\emptyset$ denotando a raiz da árvore binária considerada. Cada vértice presente na árvore 
representará um patógeno presente no sistema para o processo dos tipos. Vamos denotá-los como segue: Defina $N=\{1,2\}$ e $N^{f}=\bigcup_{i=0}^{\infty} N^{i}$, com $N^{0}=\emptyset$. Para cada $\bar{n} \in N^{f}$, defina $k(\bar{n})$ como sendo o número de coordenadas de $\bar{n}$ e $\sigma(\bar{n})=n_{1}+\ldots+n_{k(\bar{n})}, k(\emptyset)=\sigma(\emptyset)=0$. Ou seja, a primeira partícula do tipo 1 , que é posicionada no vértice à esquerda, embaixo da raiz, será denotada por $\bar{n}=1$, a segunda partícula desse mesmo tipo, que ocupa o vértice à direita abaixo da raiz, será denotada por $\bar{n}=2$. Quando a partícula $\bar{n}=2$ gerar a primeira partícula de um novo tipo, que ficará posicionada abaixo da partícula 2 , no vértice à esquerda, será identificada por $\bar{n}=(2,1)$ e assim por diante. A figura abaixo ilustra a classificação, pela definição acima, para cada vértice da árvore.

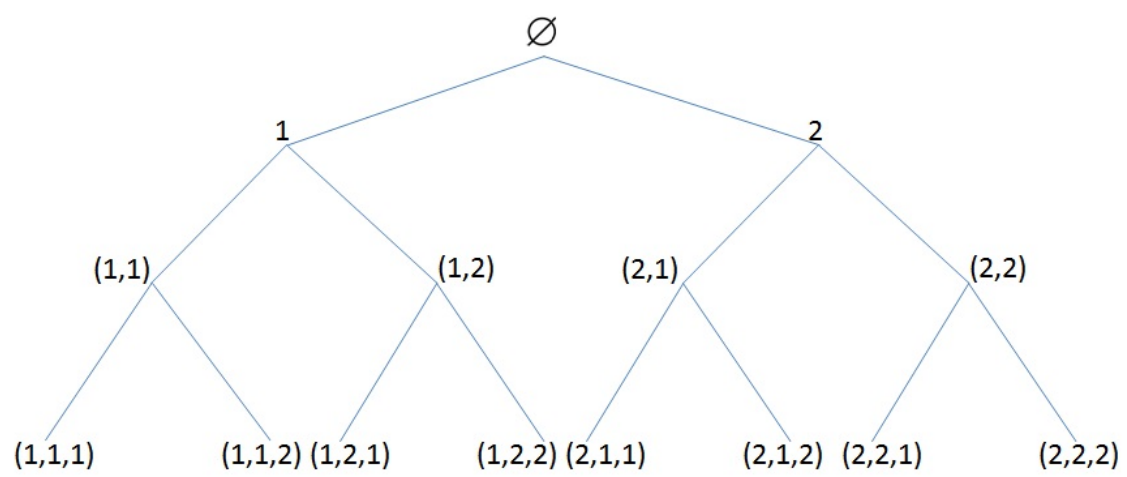

Figura 3.3: Representação da numeração de cada vértice da árvore binária até a origem do terceiro tipo.

Como queremos determinar condições para a extinção do processo, observe que se o valor esperado do número de patógenos for finito, temos que o processo evolutivo considerado será extinto. Então,

$$
\mathrm{n}^{\mathrm{o}} \text { de patógenos nascidos }=\sum_{\bar{n}} \mathbb{I}_{\{\mathrm{o} \text { patógeno } \bar{n} \text { nascer }\}}
$$

onde $\mathbb{I}_{\{A\}}(x)=\left\{\begin{array}{l}1, \quad \text { se } x \in A \\ 0, \quad \text { caso contrário. }\end{array}\right.$

Segue que,

$$
\mathbb{E}\left(\mathrm{n}^{\mathrm{o}} \text { de patógenos nascidos }\right)=\sum_{\bar{n}} \mathbb{P}(\text { o patógeno } \bar{n} \text { nascer }) .
$$

Se $\bar{n}=\left(n_{1}, \ldots, n_{k}\right)$, onde $n_{i} \in N, \forall i=1,2, \ldots, k$, o ancestral da i-ésima geração deve ter tido pelo menos $n_{i}$ descendentes. Assim,

$$
\begin{aligned}
\mathbb{P}(\text { o patógeno } \bar{n} \text { nascer }) & =\mathbb{P}\left(\sum_{i=1}^{j} \sum_{\nu=1}^{n_{i}} B_{\nu i}<\sum_{i=1}^{j} K_{i}, j=1,2, \ldots, k(\bar{n})\right) \\
& \leq \mathbb{P}\left(\sum_{i=1}^{k(\bar{n})} \sum_{\nu=1}^{n_{i}} B_{\nu i}<\sum_{i=1}^{k(\bar{n})} K_{i}\right) .
\end{aligned}
$$


Temos,

$$
\begin{aligned}
& \sum_{\bar{n}} \mathbb{P}(\text { o patógeno } \bar{n} \text { nasceu }) \leq \sum_{\bar{n}} \mathbb{P}\left(\sum_{i=1}^{k(\bar{n})} \sum_{\nu=1}^{n_{i}} B_{\nu i}<\sum_{i=1}^{k(\bar{n})} K_{i}\right) \\
& =\sum_{\bar{n}} \mathbb{P}\left(\sum_{i=1}^{k(\bar{n})} K_{i}-\sum_{i=1}^{k(\bar{n})} \sum_{\nu=1}^{n_{i}} B_{\nu i}>0\right) \\
& \leq \sum_{\bar{n}} \mathbb{E}\left[\exp \left\{u\left(\sum_{i=1}^{k(\bar{n})} K_{i}-\sum_{i=1}^{k(\bar{n})} \sum_{\nu=1}^{n_{i}} B_{\nu i}\right)\right\}\right], u>0 \\
& =\sum_{\bar{n}} \mathbb{E}\left[e^{u \sum_{i=1}^{k(\bar{n})} K_{i}}\right] \mathbb{E}\left[e^{-u \sum_{i=1}^{k(\bar{n})} \sum_{\nu=1}^{n_{i}} B_{\nu i}}\right] \\
& =\sum_{\bar{n}}\left[\left(\prod_{i=1}^{k(\bar{n})} \mathbb{E}\left(e^{u K_{i}}\right)\right)\left(\prod_{i=1}^{k(\bar{n})} \prod_{\nu=1}^{n_{i}} \mathbb{E}\left(e^{-u B_{\nu i}}\right)\right)\right] \\
& =\sum_{\bar{n}}\left[\left(\frac{1}{1-u}\right)^{k(\bar{n})} \prod_{i=1}^{k(\bar{n})}\left(\frac{\lambda r}{\lambda r+u}\right)^{n_{i}}\right], 0<u<1 \\
& =\sum_{\bar{n}}\left[\left(\frac{1}{1-u}\right)^{k(\bar{n})}\left(\frac{\lambda r}{\lambda r+u}\right)^{\sigma(\bar{n})}\right] \\
& =\sum_{k=1}^{\infty}\left[\sum_{k(\bar{n})=k}\left(\frac{1}{1-u}\right)^{k}\left(\frac{\lambda r}{\lambda r+u}\right)^{\sigma(\bar{n})}\right] \\
& =\sum_{k=1}^{\infty}\left(\frac{1}{1-u}\right)^{k}\left[\sum_{k(\bar{n})=k}\left(\frac{\lambda r}{\lambda r+u}\right)^{\sigma(\bar{n})}\right] \text {, }
\end{aligned}
$$

onde a desigualdade (3.6) é obtida pela Desigualdade Exponencial de Chebyshev que é dada por

$$
\mathbb{P}[X>a] \leq e^{(-u a)} \mathbb{E}\left[e^{u X}\right]
$$

Observe que $\sigma(\bar{n}) \leq 2 k$, se $k(\bar{n})=k$. Assim,

$$
\begin{aligned}
\sum_{k(\bar{n})=k}\left(\frac{\lambda r}{\lambda r+u}\right)^{\sigma(\bar{n})} & =\sum_{i=0}^{k}\left(\begin{array}{c}
k \\
i
\end{array}\right)\left(\frac{\lambda r}{\lambda r+u}\right)^{k+i} \\
& =\left(\frac{\lambda r}{\lambda r+u}\right)^{k} \sum_{i=0}^{k}\left(\begin{array}{c}
k \\
i
\end{array}\right)\left(\frac{\lambda r}{\lambda r+u}\right)^{i}
\end{aligned}
$$




$$
\begin{aligned}
& =\left(\frac{\lambda r}{\lambda r+u}\right)^{k}\left(\frac{\lambda r}{\lambda r+u}+1\right)^{k} \\
& =\left[\left(\frac{\lambda r}{\lambda r+u}\right)+\left(\frac{\lambda r}{\lambda r+u}\right)^{2}\right]^{k}
\end{aligned}
$$

Unindo a desigualdade (3.7) com a igualdade (3.8) acima, temos

$$
\mathbb{E}\left(\mathrm{n}^{\mathrm{o}} \text { de patógenos nascidos }\right) \leq \sum_{k=1}^{\infty}\left[\left(\frac{1}{1-u}\right)\left(\frac{\lambda r}{\lambda r+u}+\left(\frac{\lambda r}{\lambda r+u}\right)^{2}\right)\right]^{k}
$$

que será finita se

$$
\min _{0<u<1}\left\{\frac{1}{1-u}\left[\frac{\lambda r}{\lambda r+u}+\left(\frac{\lambda r}{\lambda r+u}\right)^{2}\right]\right\}<1 .
$$

Para provar a segunda parte do teorema vamos, primeiramente provar o seguinte lema.

Lema 3.1.1. Se

$$
\min _{0<u<1}\left\{\frac{1}{1-u}\left[\frac{\lambda r}{\lambda r+u}+\left(\frac{\lambda r}{\lambda r+u}\right)^{2}\right]\right\}>1,
$$

então existe $k$ tal que $\sum_{k(\bar{n})=k} \mathbb{P}$ (o patógeno $\bar{n}$ nascer $)>1$.

Prova.Seja $N_{1}, N_{2}, \ldots$ variáveis aleatórias independentes e identicamente distribuídas com $\mathbb{P}(N=1)=m$ e $\mathbb{P}(N=2)=1-m$. Sejam, ainda, $N_{1}, N_{2}, \ldots,\left\{K_{j}\right\}$ e $\left\{B_{\nu j}\right\}$ mutuamente independentes. Para $n=1,2, \ldots$ defina

$$
Z_{n}:=K_{n}-\sum_{\nu=1}^{N_{n}} B_{\nu n}
$$

Temos que $\left\{Z_{n}\right\}$ é uma sequência de variáveis aleatórias e

$$
\mathbb{E}\left(e^{u Z}\right)=\frac{1}{1-u}\left[\frac{\lambda r}{\lambda r+u} m+\left(\frac{\lambda r}{\lambda r+u}\right)^{2}(1-m)\right]
$$

De fato,

$$
\begin{aligned}
\mathbb{E}\left[e^{u Z}\right] & =\mathbb{E}\left[e^{u\left(K_{1}-\sum_{\nu=1}^{N_{1}} B_{1 \nu}\right)}\right] \\
& =\mathbb{E}\left[e^{u K_{1}}\right] \mathbb{E}\left[e^{-u \sum_{\nu=1}^{N_{1}} B_{1 \nu}}\right] \\
& =\frac{1}{1-u} \mathbb{E}\left[\prod_{\nu=1}^{N_{1}} e^{-u B_{1 \nu}}\right], 0<u<1 \\
& =\frac{1}{1-u} \mathbb{E}\left[\mathbb{E}\left[\prod_{\nu=1}^{N_{1}} e^{-u B_{1 \nu}}\right] \mid N_{1}\right]
\end{aligned}
$$




$$
\begin{aligned}
& =\frac{1}{1-u} \mathbb{E}\left[\left(\frac{\lambda r}{\lambda r+u}\right)^{N_{1}}\right] \\
& =\frac{1}{1-u}\left[\frac{\lambda r}{\lambda r+u} m+\frac{(\lambda r)^{2}}{(\lambda r+u)^{2}}(1-m)\right], \forall n \in\{1,2, \ldots\} .
\end{aligned}
$$

Assim,

$$
\begin{aligned}
& \mathbb{P}\left(\sum_{i=1}^{j} Z_{i}>0, j=1, \ldots, k\right)= \\
= & \sum_{k(\bar{n})=k} \mathbb{P}\left(\sum_{i=1}^{j} Z_{i}>0, j=1, \ldots, k \mid\left(N_{1}, \ldots, N_{k}\right)=\left(n_{1}, \ldots, n_{k}\right)\right) \mathbb{P}\left[\left(N_{1}, \ldots, N_{k}\right)=\left(n_{1}, \ldots, n_{k}\right)\right] \\
= & \sum_{k(\bar{n})=k} \mathbb{P}\left(\sum_{i=1}^{j} \sum_{\nu=1}^{n_{i}} B_{\nu i}<\sum_{i=1}^{j} K_{j}, j=1, \ldots, k\right) \mathbb{P}\left(N_{1}=n_{1}\right) \ldots \mathbb{P}\left(N_{k}=n_{k}\right) \\
= & \sum_{k(\bar{n})=k} \mathbb{P}(\text { patógeno } \bar{n} \text { ter nascido }) m^{\# 1}(1-m)^{\# 2}
\end{aligned}
$$

onde a última igualdade segue da equação (3.5) e \#i indica o número de variáveis que assumem o valor i, $i=1,2$ e $\# 1+\# 2=k$.

Se

$$
\min _{0<u<1}\left\{\frac{1}{1-u}\left[\frac{\lambda r}{\lambda r+u}+\left(\frac{\lambda r}{\lambda r+u}\right)^{2}\right]\right\}=1+\epsilon,
$$

podemos escolher $\epsilon$ suficientemente pequeno de modo que

$$
\begin{aligned}
m^{-1} \min _{0<u<1} \mathbb{E}\left[e^{u Z}\right] & =\min _{0<u<1}\left\{\frac{1}{1-u}\left[\left(\frac{\lambda r}{\lambda r+u}\right) m+\left(\frac{\lambda r}{\lambda r+u}\right)^{2}(1-m)\right]\right\} m^{-1} \\
& =\min _{0<u<1}\left\{\frac{1}{1-u}\left[\left(\frac{\lambda r}{\lambda r+u}\right)+\left(\frac{\lambda r}{\lambda r+u}\right)^{2} \frac{1-m}{m}\right]\right\} \\
& >\min _{0<u<1}\left\{\frac{1}{1-u}\left[\left(\frac{\lambda r}{\lambda r+u}\right)+\left(\frac{\lambda r}{\lambda r+u}\right)^{2}\right]\right\}, \text { se } m<1 / 2 \\
& =1+\epsilon .
\end{aligned}
$$

Agora, obseve que

$$
\begin{aligned}
\mathbb{E}[Z] & =\mathbb{E}\left[K_{i}-\sum_{\nu=1}^{N_{i}} B_{\nu i}\right]=\mathbb{E}\left[K_{i}\right]-\mathbb{E}\left[\mathbb{E}\left(\sum_{\nu=1}^{N_{i}} B_{\nu i} \mid N_{i}=n\right)\right] \\
& =1-\mathbb{E}\left[N_{i} \frac{1}{\lambda r}\right]=1-\frac{1}{\lambda r} \mathbb{E}\left[N_{i}\right] \\
& =1-\frac{1}{\lambda r}(2-m) .
\end{aligned}
$$


Logo, para $m$ suficientemente pequeno e $\lambda r<2$ temos que $\mathbb{E}(Z)<0$.

Então, pelo Lema C.4.1 (ver apêndice), para $k$ suficientemente grande temos

$$
\begin{aligned}
\mathbb{P}\left(\sum_{i=1}^{k} Z_{i}>0, k=1,2, \ldots, n\right) & =\min _{0<u<1}\left\{\frac{1}{1-u}\left[\left(\frac{\lambda r}{\lambda r+u}\right) m+\left(\frac{\lambda r}{\lambda r+u}\right)^{2}(1-m)\right]\right\} \\
& >[m(1+\epsilon)]^{k} .
\end{aligned}
$$

Assim,

$$
\sum_{k(\bar{n})=k} \mathbb{P}(\bar{n} \text { ter nascido }) m^{\# 1}(1-m)^{\# 2}>[m(1+\epsilon)]^{k} .
$$

Observe, ainda, que

$$
\sum_{k(\bar{n})=k} \mathbb{P}(\bar{n} \text { ter nascido })(1-m)^{\# 1}(1-m)^{\# 2}>\sum_{k(\bar{n})=k} \mathbb{P}(\bar{n} \text { ter nascido }) m^{\# 1}(1-m)^{\# 2}
$$

pois $m<(1-m)$ e $m^{\# 1}<(1-m)^{\# 1}$. Deste modo,

$$
\begin{aligned}
\frac{\sum_{k(\bar{n})=k} \mathbb{P}(\bar{n} \text { ter nascido })(1-m)^{\# 1}(1-m)^{\# 2}}{m^{k}} & >\frac{\sum_{k(\bar{n})=k} \mathbb{P}(\bar{n} \text { ter nascido }) m^{\# 1}(1-m)^{\# 2}}{m^{k}}, \\
& >(1+\epsilon)^{k} .
\end{aligned}
$$

onde a última desigualdade foi obtida em (3.11).

Por fim, temos que

$$
\sum_{k(\bar{n})=k} \mathbb{P}(\bar{n} \text { ter nascido })>(1+\epsilon)^{k}\left(\frac{m}{1-m}\right)^{k}
$$

aplicando o limite quando $m$ tende a $1 / 2$ em ambos os lados da desigualdade temos

$$
\sum_{k(\bar{n})=k} \mathbb{P}(\bar{n} \text { ter nascido })>1,
$$

para $k$ suficientemente grande, como queríamos provar.

Para finalizarmos a prova do critério de sobrevivência, vamos dizer que uma partícula no processo é especial se ela é a partícula mais ancestral (ou seja, a raiz), ou se ela é uma partícula da nk-ésima geração que não possui ancestrais vivos.

Temos que o número esperado de partículas na geração nk, pela desigualdade (3.12), é maior que 1. Logo, o processo das partículas especiais é um processo de ramificação supercrítico. Então, com probabilidade positiva, o processo nunca será extinto, implicando que há uma probabilidade positiva para que o processo original, considerando todas as partículas possa nunca terminar. 
Conseguimos aproximar o valor de

$$
\lambda_{c}=\min _{0<u<1}\left\{\frac{1}{1-u}\left[\frac{\lambda r}{\lambda r+u}+\left(\frac{\lambda r}{\lambda r+u}\right)^{2}\right]\right\}
$$

como segue. Queremos determinar $u \in(0,1)$ tal que

$$
\min _{0<u<1}\left\{\frac{1}{1-u}\left[\frac{\lambda r}{\lambda r+u}+\left(\frac{\lambda r}{\lambda r+u}\right)^{2}\right]\right\}<1
$$

para que possamos determinar $\lambda$, em função de $u$, de modo que possamos garantir a extinção.

Desse modo, defina

$$
f(u)=\frac{1}{1-u}\left(\frac{\lambda r}{\lambda r+u}+\left(\frac{\lambda r}{\lambda r+u}\right)^{2}\right)=\frac{1}{1-u}\left(\frac{2(\lambda r)^{2}+\lambda r u}{(\lambda r+u)^{2}}\right)
$$

onde $u \in(0,1)$. Temos que

$$
\begin{aligned}
f^{\prime}(u) & =\frac{\left[2(\lambda r)^{2}+\lambda r u\right]^{\prime}\left[(1-u)(u+\lambda r)^{2}\right]-\left[2(\lambda r)^{2}+\lambda r u\right]\left[(1-u)(u+\lambda r)^{2}\right]^{\prime}}{\left[(1-u)(u+\lambda r)^{2}\right]^{2}} \\
& =\frac{\lambda r(1-u)(u+\lambda r)^{2}-\lambda r(2 \lambda r+u)[2(1+u)(u+\lambda r)-(u+\lambda r)]}{(1-u)^{2}(u+\lambda r)^{4}} \\
& =\frac{\lambda r\left(2 u^{2}+\lambda r(-3+2 \lambda r)+u(6 \lambda r-1)\right.}{(u-1)^{2}(u+\lambda r)^{3}} .
\end{aligned}
$$

Logo,

$$
f^{\prime}(u)=0 \Leftrightarrow u=\left\{\begin{array}{l}
\frac{1}{4}\left(1-6 \lambda r-\sqrt{1+12 \lambda r+20(\lambda r)^{2}}\right) \\
\frac{1}{4}\left(1-6 \lambda r+\sqrt{1+12 \lambda r+20(\lambda r)^{2}}\right)
\end{array} .\right.
$$

Como $u \in(0,1)$, segue que

$$
\bar{u}=\frac{1}{4}\left(1-6 \lambda r+\sqrt{1+12 \lambda r+20(\lambda r)^{2}}\right),
$$

é ponto crítico de $f$, com $\lambda r \in(0,1,5)$, como mostra o gráfico abaixo.

Para garantirmos que $\bar{u}$ é ponto de mínimo, basta mostrar que $f^{\prime \prime}(\bar{u})>0$. De fato, segue que

$f^{\prime \prime}(u)=-\frac{1}{(u-1)^{3}} 2 \lambda r(\lambda r+u)^{2}\left[2(\lambda r)^{3}+(\lambda r)^{2}(3+2 u)+\lambda r\left(-5+15 u-6 u^{2}\right)+u\left(-2+6 u-3 u^{2}\right)\right]$. 


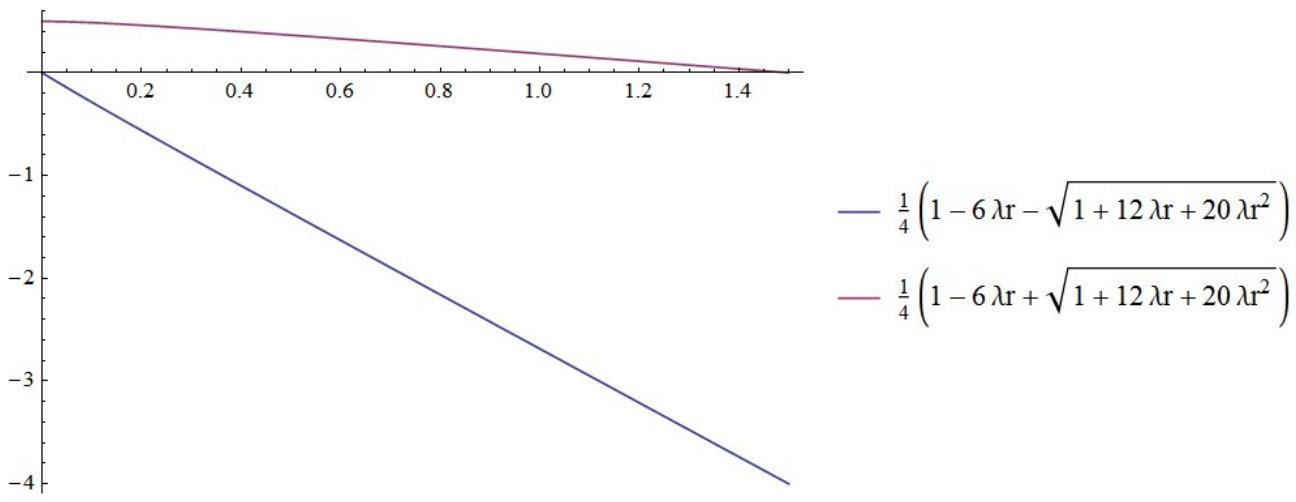

Figura 3.4: Gráfico das funções $g(\lambda r)=\frac{1}{4}\left(1-6 \lambda r-\sqrt{1+12 \lambda r+20(\lambda r)^{2}}\right)$ e $h(\lambda r)=\frac{1}{4}\left(1-6 \lambda r-\sqrt{1+12 \lambda r+20(\lambda r)^{2}}\right)$, com $0<\lambda r<1,5$.

Logo,

$$
f^{\prime \prime}(\bar{u})=\frac{\lambda \sqrt{1+12 \lambda r+20(\lambda r)^{2}}\left(1-2 \lambda r+\sqrt{1+12 \lambda r+20(\lambda r)^{2}}\right)^{3}}{4\left(-3-6 \lambda r+\sqrt{1+12 \lambda r+20(\lambda r)^{2}}\right)^{2}} .
$$

Assim, perceba que

$$
\begin{aligned}
f^{\prime \prime}(\bar{u})>0 & \Longleftrightarrow 1-2 \lambda r+\sqrt{1+12 \lambda r+20(\lambda r)^{2}}>0 \\
& \Longleftrightarrow \sqrt{1+12 \lambda r+20(\lambda r)^{2}}>2 \lambda r-1 \\
& \Longleftrightarrow 1+12 \lambda r+20(\lambda r)^{2}>(2 \lambda r-1)^{2} \\
& \Longleftrightarrow 16 \lambda r(\lambda r+1)>0 \\
& \Longleftrightarrow \lambda r>0 .
\end{aligned}
$$

Logo, f assume valor mínimo em $\bar{u}$ e, perceba que em (3.14) usamos o fato que $\lambda r>0$.

Desse modo, temos que,

$$
\begin{aligned}
f(\bar{u}) & =\min _{0<u<1}\left\{\frac{1}{1-u}\left[\frac{\lambda r}{\lambda r+u}+\left(\frac{\lambda r}{\lambda r+u}\right)^{2}\right]\right\} \\
& =\frac{16 \lambda r\left(1+2 \lambda r+\sqrt{1+12 \lambda r+20(\lambda r)^{2}}\right.}{\left[3+6 \lambda r-\sqrt{1+12 \lambda r+20(\lambda r)^{2}}\right]\left[1-2 \lambda r+\sqrt{1+12 \lambda r+20(\lambda r)^{2}}\right]^{2}}
\end{aligned}
$$

e $f(\bar{u})<1$ se, e somente se,

$$
\begin{aligned}
& \frac{16 \lambda r\left(1+2 \lambda r+\sqrt{1+12 \lambda r+20(\lambda r)^{2}}\right.}{\left[3+6 \lambda r-\sqrt{1+12 \lambda r+20(\lambda r)^{2}}\right]\left[1-2 \lambda r+\sqrt{1+12 \lambda r+20(\lambda r)^{2}}\right]^{2}}<1 \\
& \Leftrightarrow(\lambda r)^{4}-60(\lambda r)^{3}-48(\lambda r)^{2}+2 \lambda r+5>0
\end{aligned}
$$


Observe que $\lambda r=-1 / 2$ é raiz do polinômio acima. Logo, basta determinar condições sobre $\lambda r$ de modo que

$$
4(\lambda r)^{3}-32(\lambda r)^{2}-8 \lambda r+5>0
$$

Logo, temos que a condição acima é satisfeita $\forall \lambda r>0$ tal que $\lambda r<0,29$.

\subsection{Processo de infecção para o caso aleatório: influência da aleatoriedade no preenchimento da árvore para a evolução da infecção}

Na seção anterior consideramos um modelo onde os tempos de nascimento e morte são aleatórios, no entanto, a posição ocupada por cada partícula na árvore é determinística. Nesta seção propomos uma variação do modelo, de modo a ter aleatoriedade no preenchimento de cada nível da árvore. Nosso intuito é analisar se o comportamento aleatório do preenchimento exerce alguma influência para que uma infecção se torne extinta. A variação é dada como segue: com probabilidade 1/2 a mutação ocupará o vértice à esquerda imediatamente abaixo da espécie mãe e, com probabilidade $1 / 2$ o vértice da árvore binária ocupado será o da direita. Se um dos vértices já estiver ocupado, a próxima mutação ocupará o vértice vazio com probabilidade 1 .

\subsubsection{Análise de sobrevivência e extinção considerando um ramo fixo}

Assim como a análise apresentada na seção 3.1.1, queremos estudar condições sobre os parâmetros para a sobrevivência e a extinção do processo quando fixamos um ramo.

No modelo sem aleatoriedade, para a ocupação da árvore temos, pelo Teorema 3.1.1, que independentemente do ramo escolhido, se $\lambda \leq 1 / r$ o processo sobre o ramo é extinto com probabilidade 1 e, se $\lambda>\frac{1+\sqrt{2}}{r}$ o processo sobrevive com probabilidade positiva.

Como mostra o teorema abaixo, para o caso em que há aleatoriedade no preenchimento da árvore, provamos a existência de um único ponto crítico para todos os ramos.

Teorema 3.2.1. Seja $\lambda>0$ e fixe $r, 0<r<1$. Existe $\lambda_{c}=\frac{1+\sqrt{5}}{2 r}$ tal que, se $\lambda \leq \lambda_{c}$ então o processo de evolução será extinto com probabilidade 1 e, se $\lambda>\lambda_{c}$, então o processo de evolução sobrevive com probabilidade positiva.

Observação 3.2.1. Antes de provarmos o resultado, perceba que como há uma da dinâmica aleatória para o preenchimento da árvore, se fixarmos o ramo extremo à direita e $\bar{n}=(1,1)$ temos, uma vez que a partícula $\bar{n}=1$ já tenha nascido, os seguintes acontecimentos fazem com que tenhamos uma partícula nesta posição.

Pela Figura 3.5, temos que há duas formas para que o vértice $(1,1)$ da árvore seja ocupado. Sendo $B_{i}$, variáveis aleatórias com distribuição exponencial de parâmetro $\lambda r$ e $K_{i}$ 


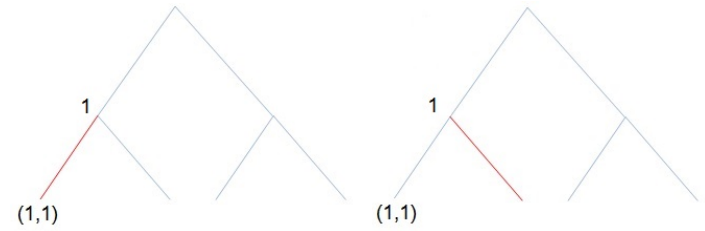

Figura 3.5: Representação das formas de como uma partícula pode ocupar a posição $\bar{n}=(1,1)$, uma vez que a partícula $\bar{n}=1$ já nasceu. Os traços em vermelho representam a primeira mutação ocorrida.

exponenciais de parâmetro 1, temos que a primeira possibilidade se dá quando a primeira mutação escolhe aquele vértice, e isso ocorre com probabilidade $\frac{1}{2} \mathbb{P}\left(B_{1}<K_{1}\right)$. Já a segunda possibilidade, é quando a segunda mutação ocupa o vértice e tal evento ocorre com probabilidade $\frac{1}{2} \mathbb{P}\left(B_{1}<K_{1}, B_{1}+B_{2}<K_{1}\right)$

Prova.Seguindo as ideias apresentadas na prova do Teorema 3.1.1, defina $X(t)$ como sendo o número de patógenos no ramo em um instante de tempo $t \geq 0$, onde $X(0)=1$. Temos que

$$
p_{i}:=\mathbb{P}(X(t+s)=k+1 \mid X(t)=k)=\frac{1}{2}\left[\mathbb{P}\left(B_{1}<K_{1}\right)+\mathbb{P}\left(B_{1}<K_{1}, B_{1}+B_{2}<K_{1}\right)\right]=: \alpha
$$

$\mathrm{e}$

$$
q_{i}=\mathbb{P}(X(t+s)=k-1 \mid X(t)=k)=1-\mathbb{P}(X(t+s)=k+1 \mid X(t)=k)=: 1-\alpha .
$$

Observe que uma vez que temos $k$ indivíduos no ramo, para que essa quantidade aumente em uma unidade, é necessário que ocorra o nascimento de uma mutação no ramo, antes de um tempo de morte. Esse indivíduo pode surgir de duas formas, sendo o primeiro filho ou o segundo. Tome

$$
T_{0}:=\inf \{t: X(t)=2\}
$$

ou seja, o instante de tempo no qual surge o segundo tipo no ramo escolhido. Usando os mesmos argumentos apresentados na prova do Teorema 3.1.1, temos que o processo será recorrente quando

$$
\sum_{i=1}^{\infty}\left(\frac{1-\alpha}{\alpha}\right)^{i}=+\infty
$$

caso contrário ele será transiente.

Temos que

$$
\begin{aligned}
\alpha & =\mathbb{P}(X(t+s)=k+1 \mid X(t)=k)=\frac{1}{2}\left[\mathbb{P}\left(B_{1}<K_{1}\right)+\mathbb{P}\left(B_{1}<K_{1}, B_{1}+B_{2}<K_{1}\right)\right] \\
& =\frac{1}{2}\left[\frac{\lambda r}{1+\lambda r}+\mathbb{P}\left(B_{1}+B_{2}<K_{1}\right)\right]
\end{aligned}
$$




$$
\begin{aligned}
& =\frac{1}{2}\left[\frac{\lambda r}{1+\lambda r}+\mathbb{P}\left(B_{1}+B_{2}-K_{1}<0\right)\right] \\
& =\frac{1}{2}\left[\frac{\lambda r}{1+\lambda r}+\mathbb{P}\left(G-K_{1}<0\right)\right] .
\end{aligned}
$$

Observe que na igualdade (3.17) foi usado o fato que $\left\{B_{1}+B_{2}<K_{1}\right\} \subset\left\{B_{1}<K_{1}\right\}$, isto é, $\mathbb{P}\left(B_{1}<K_{1}, B_{1}+B_{2}<K_{1}\right)=\mathbb{P}\left(B_{1}+B_{2}<K_{1}\right)$ e em (3.17) temos $G:=B_{1}+B_{2}$, onde $G$ tem distribuição Gama de parâmetros 2 e $\lambda r$.

Por fim, para obtermos o valor de $\alpha$, basta determinar $\mathbb{P}\left(G-K_{1}<0\right)$. Temos,

$$
\mathbb{P}\left(G-K_{1}<0\right)=\int_{-\infty}^{0} \int_{0}^{+\infty} f_{G}(x) f_{K_{1}}(x-z) d x d z,
$$

onde $f_{G}$ e $f_{K_{1}}$ é a função densidade de probabilidade de $G$ e de $K_{1}$, respectivamente.

Logo,

$$
\begin{aligned}
\mathbb{P}\left(G-K_{1}<0\right) & =\int_{-\infty}^{0} \int_{0}^{+\infty} f_{G}(x) f_{K_{1}}(x-z) d x d z \\
& =\int_{-\infty}^{0} \int_{0}^{+\infty} \frac{(\lambda r)^{2}}{\Gamma(2)} e^{-\lambda r x} x e^{-(x-z)} d x d z \\
& =\int_{-\infty}^{0} \frac{(\lambda r)^{2}}{(\lambda r+1)^{2}} e^{z}\left[\int_{0}^{+\infty} \frac{(1+\lambda r)^{2}}{\Gamma(2)} e^{-(1+\lambda r) x} x d x\right] d z \\
& =\int_{-\infty}^{0} \frac{(\lambda r)^{2}}{(\lambda r+1)^{2}} e^{z} d z
\end{aligned}
$$

Observe que em (3.18) temos que $\frac{(1+\lambda r)^{2}}{\Gamma(2)} e^{-(1+\lambda r) x} x$ é a função densidade de probabilidade de uma variável aleatória Gama de parâmetros 2 e $1+\lambda r$, por isso a integral vale 1.

Por fim,

$$
\begin{aligned}
\int_{-\infty}^{0} \frac{(\lambda r)^{2}}{(\lambda r+1)^{2}} e^{z} d z & =\frac{(\lambda r)^{2}}{(\lambda r+1)^{2}} \int_{-\infty}^{0} e^{z} d z \\
& =\frac{(\lambda r)^{2}}{(\lambda r+1)^{2}}
\end{aligned}
$$

uma vez que $\int_{-\infty}^{0} e^{z} d z=1$

Assim, por (3.17) e (3.19), temos que

$$
\alpha=\frac{1}{2}\left[\frac{\lambda r}{1+\lambda r}+\left(\frac{\lambda r}{1+\lambda r}\right)^{2}\right] .
$$


Desse modo,

$$
\begin{aligned}
q_{i} & =1-\alpha \\
& =1-\frac{1}{2}\left[\frac{\lambda r}{1+\lambda r}+\left(\frac{\lambda r}{1+\lambda r}\right)^{2}\right] \\
& =\frac{2+3 \lambda r}{2(1+\lambda r)^{2}}
\end{aligned}
$$

Portanto, o processo será recorrente se, e somente se, $\sum_{i=1}^{\infty}\left(\frac{1-\alpha}{\alpha}\right)^{i}=+\infty$. Ou seja, se

$$
\begin{aligned}
\frac{1-\alpha}{\alpha} \geq 1 & \Leftrightarrow \frac{\frac{2+3 \lambda r}{2(1+\lambda r)^{2}}}{\frac{1}{2}\left[\frac{\lambda r}{1+\lambda r}+\left(\frac{\lambda r}{1+\lambda r}\right)^{2}\right]} \geq 1 \\
& \Leftrightarrow \frac{2+3 \lambda r}{2(\lambda r)^{2}+\lambda r} \geq 1
\end{aligned}
$$

Assim, queremos encontrar $\lambda r$ tal que

$$
2+3 \lambda r \geq 2(\lambda r)^{2}+\lambda r
$$

Então,

$$
2+3 \lambda r \geq 2(\lambda r)^{2}+\lambda r \Leftrightarrow-2(\lambda r)^{2}+2(\lambda r)+2 \geq 0
$$

Por fim, basta encontrar a raiz da função $f(\lambda r)=-2(\lambda r)^{2}+2(\lambda r)+2$ e analisar para quais valores ela é não negativa.

Temos que

$$
f(\lambda r)=0 \Leftrightarrow \lambda r=\frac{1 \pm \sqrt{5}}{2} .
$$

Portanto, se $0<\lambda r \leq \frac{1+\sqrt{5}}{2}$, temos que o processo sobre o ramo é recorrente e, portanto,

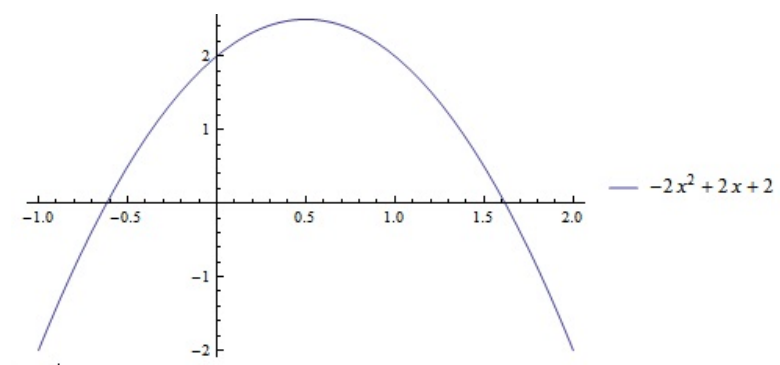

Figura 3.6: Gráfico da função $f(x)=-2 x^{2}+2 x+2$, com $-1<x<2$.

será extinto com probabilidade 1 . Mas, se $\lambda r>\frac{1+\sqrt{5}}{2}$, então o processo é transiente e com probabilidade positiva a infecção sobrevive para sempre no ramo.

Observação 3.2.2. Se com probabilidade $p \in[0,1]$ o novo patógeno escolhe o ramo à 
esquerda, temos que $\lambda_{c}=\frac{1-p+\sqrt{p^{2}-2 p+2}}{r}$ pois, neste caso,

$$
\alpha=p \mathbb{P}\left(B_{1}<K_{1}\right)+(1-p) \mathbb{P}\left(B_{1}<K_{1}, B_{1}+B_{2}<K_{1}\right)=\frac{(\lambda r)^{2}+p \lambda r}{(1+\lambda r)^{2}}
$$

$e$

$$
1-\alpha=\frac{1+(2-p) \lambda r}{(1+\lambda r)^{2}} .
$$

Assim, pelos mesmos argumentos apresentados na prova do Teorema 3.2.1 temos que

$$
\sum_{i=1}^{+\infty}\left(\frac{1-\alpha}{\alpha}\right)^{i}=+\infty \Leftrightarrow-(\lambda r)^{2}+(2-2 p) \lambda r+1 \geq 0
$$

ou seja, se

$$
1-p-\sqrt{p^{2}-2 p+2} \leq \lambda r \leq 1-p+\sqrt{p^{2}-2 p+2}
$$

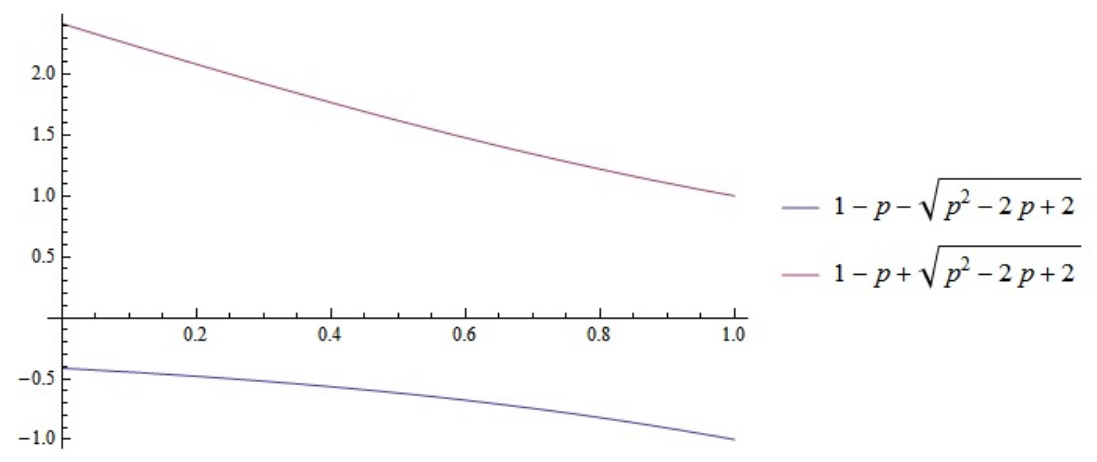

Figura 3.7: Gráfico das funções $f(p)=1-p-\sqrt{p^{2}-2 p+2}$ e $g(p)=1-p+\sqrt{p^{2}-2 p+2}$, com $0 \leq p \leq 1$.

Pelo gráfico acima temos que $f(p)<0, \forall p \in[0,1]$. Portanto, $0<\lambda r \leq 1-p+$ $\sqrt{p^{2}-2 p+2}$ para que $\sum_{i=1}^{+\infty}\left(\frac{1-\alpha}{\alpha}\right)^{i}=+\infty$

Por fim, pelos argumentos já apresentados anteriormente, temos que

$$
\lambda_{c}=\frac{1-p+\sqrt{p^{2}-2 p+2}}{r} .
$$

\subsubsection{Análise de extinção para o processo de infecção na árvore}

Vamos agora analisar a extinção na árvore como um todo e, relembre que quando não há aleatoriedade na ocupação dos vértices da árvore binária, basta que $\lambda r<0,29$ para que ocorra extinção com probabilidade 1 . O teorema abaixo nos garante que se $\lambda r<3-2 \sqrt{2}$, então temos morte do modelo com aleatoriedade. Ou seja, quando combatemos a infecção 
na árvore com preenchimento aleatório, garantimos também a extinção da infecção quando os patógenos podem preencher o grafo de modo não aleatório.

Teorema 3.2.2. Seja $\lambda>0$ e $0<r<1$. Se $\lambda r \leq 3-2 \sqrt{2}$, então o processo de evolução será extinto com probabilidade 1.

Observação 3.2.3. Antes de provarmos o resultado acima, perceba que como há uma da dinâmica aleatória para o preenchimento da árvore, se fixarmos $\bar{n}=(1,2)$ temos que os seguintes acontecimentos fazem com que tenhamos uma partícula nesta posição.
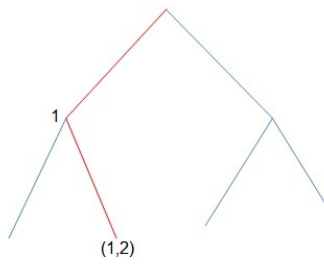

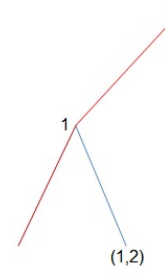

$(1,2)$

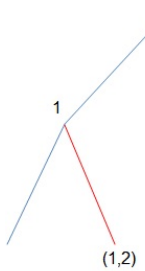

$(1,2)$

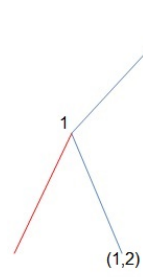

$(1,2)$

Figura 3.8: Representação das formas de como uma partícula pode ocupar a posição $\bar{n}=(1,2)$. Os traços em vermelho representam a primeira mutação ocorrida e os em azul a segunda.

As figuras acima representam todas as possibilidades que temos para que uma partícula ocupe a posição $\bar{n}=(1,2)$ no grafo considerado.

Na primeira figura, tanto o ancestral quanto a partícula na posição $\bar{n}$ foram as primeiras mutações. Logo,

$$
\begin{aligned}
& \mathbb{P}(\bar{n} \text { nascer } \mid \text { o ancestral e a partícula são as primeiras mutações }) \\
& =\frac{1}{4} \mathbb{P}\left(B_{\nu 1}<K_{1}, B_{\nu 1}+B_{\nu 2}<K_{1}+K_{2}\right) .
\end{aligned}
$$

No segundo caso, o ancestral da partícula foi a primeira mutação e a partícula considerada foi a segunda. Assim,

$$
\begin{aligned}
& \mathbb{P}(\bar{n} \text { nascer } \mid \text { o ancestral é a primeira mutação e a partícula é a segunda mutação }) \\
& =\frac{1}{4} \mathbb{P}\left(B_{\nu 1}<K_{1}, B_{\nu 1}+B_{\nu 2}+B_{\nu 3}<K_{1}+K_{2}\right) .
\end{aligned}
$$

Na sequência, o ancestral de $\bar{n}$ é a segunda mutação e a partícula é a primeira mutação. Então,

$$
\begin{aligned}
& \mathbb{P}(\bar{n} \text { nascer } \mid \text { o ancestral é a segunda mutação e a partícula é a primeira }) \\
& =\frac{1}{4} \mathbb{P}\left(B_{\nu 1}+B_{\nu 2}<k_{1}, B_{\nu 1}+B_{\nu 2}+B_{\nu 3}<K_{1}+K_{2}\right) .
\end{aligned}
$$

Por fim, na quarta imagem, tanto o ancestral, quanto a partícula são as segundas mutações. 
Desse modo,

$$
\begin{aligned}
& \mathbb{P}(\bar{n} \text { nascer|o ancestral e a partícula são as segundas mutações }) \\
& =\frac{1}{4} \mathbb{P}\left(B_{\nu 1}+B_{\nu 2}<k_{1}, B_{\nu 1}+B_{\nu 2}+B_{\nu 3}+B_{\nu 4}<K_{1}+K_{2}\right)
\end{aligned}
$$

Isto posto, temos que

$$
\begin{aligned}
\mathbb{P}(\bar{n}=(1,2) \text { nascer }) & =\frac{1}{4} \mathbb{P}\left(B_{\nu 1}<K_{1}, B_{\nu 1}+B_{\nu 2}<K_{1}+K_{2}\right) \\
& +\frac{1}{4} \mathbb{P}\left(B_{\nu 1}<K_{1}, B_{\nu 1}+B_{\nu 2}+B_{\nu 3}<K_{1}+K_{2}\right) \\
& +\frac{1}{4} \mathbb{P}\left(B_{\nu 1}+B_{\nu 2}<k_{1}, B_{\nu 1}+B_{\nu 2}+B_{\nu 3}<K_{1}+K_{2}\right) \\
& +\frac{1}{4} \mathbb{P}\left(B_{\nu 1}+B_{\nu 2}<k_{1}, B_{\nu 1}+B_{\nu 2}+B_{\nu 3}+B_{\nu 4}<K_{1}+K_{2}\right) \\
& \leq \mathbb{P}\left(B_{\nu 1}<K_{1}, B_{\nu 1}+B_{\nu 2}<K_{1}+K_{2}\right),
\end{aligned}
$$

onde a desigualdade (3.21) foi obtida utilizando o fato que o evento

$$
\left\{B_{\nu 1}<K_{1}, B_{\nu 1}+B_{\nu 2}<K_{1}+K_{2}\right\}
$$

é o que possui a maior probabilidade.

Podemos generalizar o que foi apresentado na observação 3.2.3 para uma partícula presente em uma posição $\bar{n} \in N^{f}$ qualquer. Tal ideia será utilizada na prova do Teorema 3.2.2.

Prova.(Teorema 3.2.2) Queremos determinar condições para a extinção do modelo proposto. Como já argumentado anteriormente, basta mostrar que

$\mathbb{E}[$ número de partículas no sistema $]<+\infty$.

Assim,

$$
\begin{aligned}
\mathbb{E}\left[\mathrm{n}^{\mathrm{o}} \text { partículas no sistema }\right] & =\mathbb{E}\left[\sum_{\bar{n}} \mathbb{I}_{\{\bar{n} \text { nascer }\}]}\right] \\
& =\sum_{\bar{n}} \mathbb{P}(\bar{n} \text { nascer }) \\
& \leq \sum_{\bar{n}} \mathbb{P}\left(\sum_{i=1}^{j} B_{i}<\sum_{i=1}^{j} K_{i}, j=1, \ldots, k(\bar{n})\right) \\
& \leq \sum_{\bar{n}} \mathbb{P}\left(\sum_{i=1}^{k(\bar{n})} B_{i}<\sum_{i=1}^{k(\bar{n})} K_{i}\right)
\end{aligned}
$$




$$
\begin{aligned}
& \leq \sum_{\bar{n}} \mathbb{E}\left[\exp \left\{u\left(\sum_{i=1}^{k(\bar{n})} K_{i}-\sum_{i=1}^{k(\bar{n})} B_{i}\right)\right\}\right] \\
& =\sum_{\bar{n}} \mathbb{E}\left[e ^ { u \sum _ { i = 1 } ^ { k ( \overline { n } } K _ { i } ] } \mathbb { E } \left[e^{\left.-u \sum_{i=1}^{k(\bar{n})} B_{i}\right]}\right.\right. \\
& =\sum_{\bar{n}} \prod_{i=1}^{k(\bar{n})}\left(\frac{1}{1-u}\right) \prod_{i=1}^{k(\bar{n})}\left(\frac{\lambda}{\lambda+u}\right), 0<u<1 \\
& =\sum_{\bar{n}}\left(\frac{1}{1-u}\right)^{k(\bar{n})}\left(\frac{\lambda}{\lambda+u}\right)^{k(\bar{n})} \\
& =\sum_{k=1}^{\infty} \sum_{k(\bar{n})=k}\left(\frac{1}{1-u}\right)^{k}\left(\frac{\lambda}{\lambda+u}\right)^{k} \\
& =\sum_{k=1}^{\infty} \sum_{i=0}^{k}\left(\begin{array}{l}
k \\
i
\end{array}\right)\left(\frac{1}{1-u}\right)^{k}\left(\frac{\lambda}{\lambda+u}\right)^{k} \\
& =\sum_{k=1}^{\infty}\left(2 \frac{1}{1-u} \frac{\lambda}{\lambda+u}\right)^{k}
\end{aligned}
$$

onde a desigualdade (3.23) foi obtida utilizando a Desigualdade Exponencial de Chebyshev.

Logo, por (3.24),

$$
\min _{0<u<1}\left\{2 \frac{1}{1-u} \frac{\lambda}{\lambda+u}\right\}<1 \Rightarrow \mathbb{E}\left[\mathrm{n}^{\mathrm{o}} \text { partículas no sistema }\right]<+\infty .
$$

Seja $f(u):=2 \frac{1}{1-u} \frac{\lambda}{\lambda+u}$, temos

$$
f^{\prime}(u)=\frac{2 \lambda(\lambda+2 u-1)}{(1-u)^{2}(\lambda+u)^{2}} .
$$

Logo, o mínimo de $f$ é obtido tomando $\bar{u}=\frac{1-\lambda}{2}$, e então

$$
\min _{0<u<1}\left\{2 \frac{1}{1-u} \frac{\lambda}{\lambda+u}\right\}=f(\bar{u})=\frac{8 \lambda}{(\lambda+1)^{2}}
$$

Por fim, temos que $f(\bar{u})<1 \Leftrightarrow \frac{8 \lambda}{(\lambda+1)^{2}}-1<\left.0\right|_{\lambda \in(0,1)}$. Ou seja, se $\lambda \in(0,3-2 \sqrt{2})$.

Assim, se $\lambda<3-2 \sqrt{2}$, temos que o processo de infecção, para o qual a ocupação dos vértices do grafo é aleatória, é extinto com probabilidade 1.

Observe o gráfico abaixo (3.9. Usando os resultados obtidos nos teoremas 3.2.1 e 3.2.2, temos que se $\lambda<\frac{3-2 \sqrt{2}}{r}$ (região $\mathrm{A}$ ), o processo de ramificação na árvore com preenchimento aleatório será extinto com probabilidade 1 . Se $\lambda>\frac{1+\sqrt{5}}{2 r}$ (região B), então o processo sobreviverá com probabilidade positiva. 


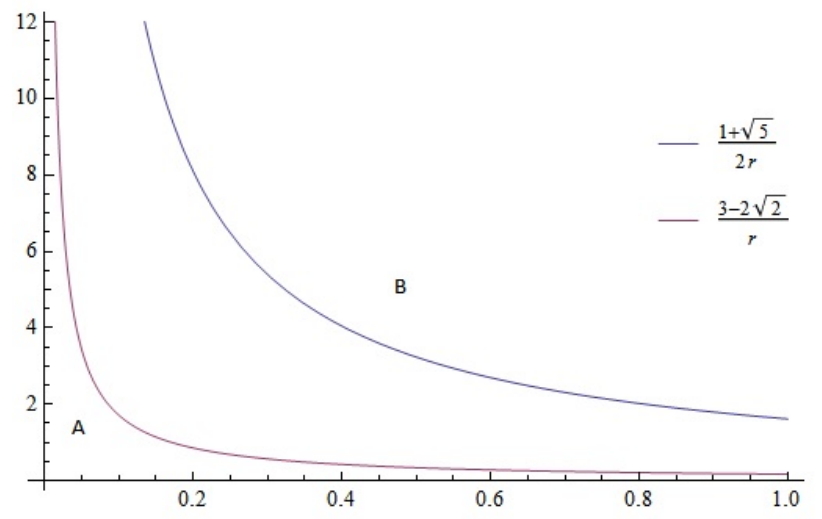

Figura 3.9: Gráfico das funções $f(r)=\frac{3-2 \sqrt{2}}{r}$ e $g(r)=\frac{1+\sqrt{5}}{2 r}$, com $0 \leq r \leq 1$. 


\section{Apêndice A}

\section{Passeio aleatório simples (PAS) em $\mathbb{Z}$}

No capítulo 2 utilizamos resultados conhecidos para o processo estocástico denominado Passeio Aleatório Simples (PAS). Nesse apêndice vamos definir tal processo e provar o resultado de recorrência que foi usado. Tais resultados são muito conhecidos na literatuda de processos estocásticos e podem ser encontrados em Bhattacharya e Waymire (2009), Ross (2009), Feller (1968), Schinazi (1999), entre outros.

A fim de dar uma idéia intuitiva sobre o processo, imagine a seguinte situação: colocamos uma partícula no sítio 0 e a cada instante discreto de tempo a partícula se move para o sítio vizinho à direita com probabilidade $p$ e, com probabilidade $1-p$ para o vizinho à esquerda. A trajetória realizada pela partícula é o que chamamos de passeio aleatório simples.

Formalmente, definimos o processo descrito acima como segue.

Definição A.0.1. Sejam $S_{1}, S_{2}, \ldots$ variáveis aleatórias independentes e identicamente distribuídas, tais que $\mathbb{P}(S=1)=p$ e $\mathbb{P}(S=-1)=1-p$. Então, a posição $X_{n}$ da partícula no instante $n \in \mathbb{N}$ é dado por

$$
X_{n}=S_{0}+S_{1}+\ldots+S_{n}, \quad S_{0}=0 .
$$

O processo estocástico $\left\{X_{n}: n \in \mathbb{N}\right\}$, definido acima, é chamado passeio aleatório simples. Quando $p=1 / 2$ ele é chamado de passeio aleatório simples e simétrico.

Observação A.0.4. Se o passeio aleatório simples começa em um sítio $x \neq 0$, temos

$$
X_{n}^{x}=x+S_{1}+\ldots+S_{n}
$$




\section{A.1 Propriedade de transiência e recorrência do pas- seio aleatório simples}

Considerando o modelo definido acima, queremos estudar há existência de transição de fase do processo.

Definição A.1.1. Definimos o instante em que o PAS, com $X_{0}=x$, atinge o sítio y pela primeira vez como sendo

$$
T_{y}^{x}=\min \left\{n \geq 1: X_{n}^{x}=y\right\} .
$$

Definição A.1.2. (Recorrência e Transiência) Dizemos que $X_{n}$ é recorrente se

$$
\mathbb{P}\left(T_{0}^{0}<+\infty\right)=1
$$

Caso contrário, $X_{n}$ será dito transiente.

Teorema A.1.1. Para $p>1 / 2$ temos que o passeio aleatório simples será transiente e para $p \leq 1 / 2$ ele é recorrente.

Prova. Seja $\phi(x)=\mathbb{P}\left(T_{y}^{x}<T_{0}^{x}\right)$, ou seja, a probabilidade do passeio aleatório simples começando em $x$ atinja o sítio $y$ ante do sítio 0 . Temos que,

$$
\phi(x)=p \phi(x+1)+(1-p) \phi(x-1) .
$$

Logo,

$$
\phi(x+1)-\phi(x)=\frac{q}{p}[\phi(x)-\phi(x-1)] .
$$

Uma vez que $\phi(0)=0$ podemos escrever $\phi(x)$ como sendo

$$
\begin{aligned}
\phi(x) & =\sum_{i=0}^{x-1}[\phi(x+1)-\phi(x)] \\
& =\sum_{i=0}^{x-1}\left(\frac{q}{p}\right)^{i} \phi(1),
\end{aligned}
$$

onde A.2 foi obtida usando A.1. Se $p=1 / 2$ temos que

$$
\phi(x)=\sum_{i=0}^{x-1} \phi(1)=x \phi(1) .
$$

Para obter $\phi(1)$ para as duas iguades acima, basta usar o fato que $\phi(y)=1$. Deste modo

$$
1=\phi(y)=y \phi(1) \Leftrightarrow \phi(1)=\frac{1}{y} \text {. }
$$


Por outro lado, se $p \neq 1 / 2$, então

$$
\begin{aligned}
\phi(x) & =\phi(1) \phi(x)=\sum_{i=0}^{x-1}\left(\frac{q^{i}}{p}\right) \\
& =\phi(1) \frac{1-\left(\frac{q}{p}\right)^{x}}{1-\frac{q}{p}} .
\end{aligned}
$$

Por argumentos análogos aos feitos em A.3, segue que

$$
1=\phi(1) \frac{1-\frac{q}{p}}{1-\left(\frac{q}{p}\right)^{y}} .
$$

Assim,

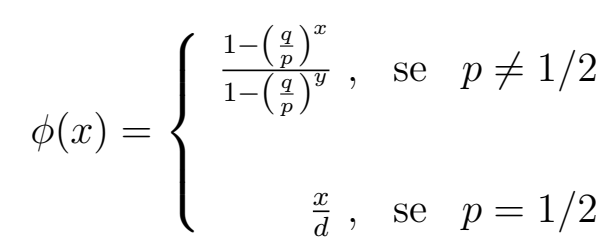

Por fim, observe que

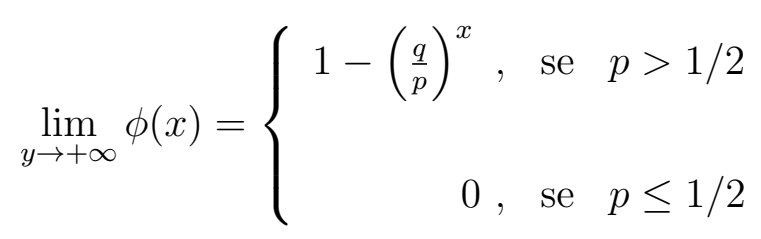

Ou seja, se $p>1 / 2$ existe uma probabilidade positiva do processo nunca atingir a origem; quando $p \leq 1 / 2$ com probabilidade 1 o processo atinge a origem. Se pensarmos no processo $\left\{X_{n}\right\}$, para $p \leq 1 / 2$, temos que com probabilidade 1 ele retorna a origem infinitas vezes isto é, o processo é recorrente - pois a cada retorno, podemos pensar que o processo está começando novamente, uma vez que ele é markoviano. Caso contrário, ele será transiente. 
APÊNDICE A 


\section{Apêndice B}

\section{Processo de ramificação}

Na prova do teorema 3.2.2 do capítulo 3 usamos o resultado de sobrevivência para um processo de ramificação. Neste apêndice definimos tal modelo e apresentamos a prova do teorema. Tal resultado pode ser encontrado facilmente na literatura de processos estocásticos e indicamos aqui algumas bibliografias: Athreya e Ney. (1972), Bhattacharya e Waymire (2009), Harris (1989), Ross (2009), entre outros.

Considere uma população composta por indivíduos capazes de gerar descendentes do mesmo tipo. Seja $G_{0}$ o tamanho da geração 0, isto é, da geração inicial e neste texto vamos supor $G_{0}=1$, ou seja, começamos nosso processo com um único indivíduo. Temos que todos os descendentes da geração 0 constituem a geração 1, e sua quantidade de indivíduos será denotada por $G_{1}$. De modo geral, $G_{n}$ denotará o tamanho da n-ésima geração. Assim, a cadeia de Markov $\left\{G_{n}, n \geq 0\right\}$ é o que chamamos de processo de ramificação.

Suponha que cada indivíduo gere ao longo de sua vida $k$ descendentes com probabilidade $P_{k}, k \geq 0$, independentemente do número de descendentes gerados por qualquer outro indivíduo. Formalmente, seja $X$ uma variável aleatória não negativa e $\left\{X_{n-1, j}, n, j \geq 1\right\}$ variáveis aleatórias independentes e identicamente distribuídas e tal que $X_{n-1, j}$ é distribuída como $X$ com $P_{k}=\mathbb{P}(X=k), \forall k \geq 0$. Observe que $X_{n j}$ representa o número de descendentes do $j$-ésimo indivíduo da geração $n-1$.

Sendo $G_{n-1}$ o tamanho da $(n-1)$-ésima geração, temos que

$$
G_{n}=\sum_{j=1}^{G_{n-1}} X_{n-1, j}, \quad n \geq 1
$$

será o tamanho da geração $G_{n}$.

Queremos estudar as probabilidades de extinção e sobrevivência do processo. Assim, definimos que o processo é extinto se, e somente se, existe $n>0$ tal que $G_{n}=0$. De modo análogo, definimos que há sobrevivência se, e somente se, $G_{n} \neq 0$ para todo $n \geq 0$.

Logo, temos que

$$
\mathbb{P}(\text { extinção })=1-\mathbb{P}(\text { sobrevivência }) \text {. }
$$


Observe que se $P_{0}=0$, temos que todos os indivíduos terão pelo menos um filho. Por outro lado, se $P_{0}=1$, então o processo se tornará extinto na geração 1. Portanto, para evitar trivialidades, vamos supor

$$
0<P_{0}<1
$$

A fim de estudar os eventos definidos anteriormente, apresentamos o teorema abaixo que nos garante condições tanto para sobrevvivência, qaunto para a extinção do processo.

Teorema B.0.2. Seja $0<P_{0}<1$ e $m=\mathbb{E}[X]$. Se $m>1$, então o processo de ramificação $\left\{G_{n}\right\}_{n \geq 0}$ sobrevive com probabilidade positiva. Caso contrário, ele será extinto com probabilidade 1.

Utilizaremos a função geradora da variável $G_{n}$ para provarmos o teorema B.0.2. Assim, antes de iniciarmos a sua demonstração, vamos definir e estudar suas propriedades.

Definição B.0.3. Seja $G_{n}$ uma variável aleatória definida em $\mathbb{N}$. A função geradora de $G_{n}$ $\left(\gamma_{n}\right)$ é dada por

$$
\gamma_{n}(s):=\mathbb{E}\left[s^{G_{n}}\right]=\sum_{k=0}^{\infty} s^{k} p_{k}, 0 \leq s \leq 1 .
$$

Lema B.0.1. Para todo $n \geq 1$ e para todo $s \in[0,1)$ temos que

$$
\begin{aligned}
\gamma_{n+1}(s) & =\gamma_{n}(\gamma(s)) \\
& =\gamma\left(\gamma_{n}(s)\right)
\end{aligned}
$$

Prova. Para provar a primeira igualdade, perceba que da definição B.0.3 segue que

$$
\gamma_{n+1}(s)=\sum_{k=0}^{\infty} s^{k} \mathbb{P}\left(G_{n+1}=k\right) .
$$

Usando B.1, temos

$$
\begin{aligned}
\mathbb{P}\left(G_{n+1}=k\right) & =\sum_{j=0}^{\infty} \mathbb{P}\left(G_{n+1}=k \mid G_{n}=j\right) \mathbb{P}\left(G_{n}=j\right) \\
& =\sum_{j=0}^{\infty} \mathbb{P}\left(X_{n, 1}+\ldots+X_{n, j}=k \mid G_{n}=j\right) \mathbb{P}\left(G_{n}=j\right) \\
& =\sum_{j=0}^{\infty} \mathbb{P}\left(X_{n, 1}+\ldots+X_{n, j}=k\right) \mathbb{P}\left(G_{n}=j\right)
\end{aligned}
$$

onde B.5 foi obtida usando o fato que as variáveis $X_{n, j}$ são independentes do enento $\left\{G_{n}=\right.$ $j\}$. 
Substituindo B.5 em B.4 temos

$$
\begin{aligned}
\gamma_{n+1}(s) & =\sum_{j=0}^{\infty} \mathbb{P}\left(G_{n}=j\right)\left[\sum_{k=0}^{\infty} s^{k} \mathbb{P}\left(X_{n, 1}+\ldots+X_{n, j}=k\right)\right] \\
& =\sum_{j=0}^{\infty} \mathbb{P}\left(G_{n}=j\right) \mathbb{E}\left[s^{X_{n, 1}+\ldots+X_{n, j}}\right] \\
& =\sum_{j=0}^{\infty} \mathbb{P}\left(G_{n}=j\right) \mathbb{E}^{j}\left[s^{X_{0,1}}\right] \\
& =\sum_{j=0}^{\infty} \mathbb{P}\left(G_{n}=j\right) \gamma_{1}^{j}(s) \\
& =\gamma_{n}(\gamma(s))
\end{aligned}
$$

Para provarmos a segunda desigualdade, basta usarmos o que acabamos de provar e iterá-lo. Logo,

$$
\gamma_{n+1}(s)=\gamma_{n}(\gamma(s))=\gamma_{n-1}\left(\gamma(\gamma(s))=\gamma_{n-1}\left(\gamma_{2}(s)\right)=\ldots=\gamma\left(\gamma_{n}(s)\right)\right.
$$

Observação B.0.1. Da definição de $\gamma$ segue que $\mathbb{P}\left(G_{n}=0\right)=\gamma_{n}(0)$.

\section{Demonstração do Teorema B.0.2}

Defina $\alpha:=\mathbb{P}($ extinção $)$ e $\alpha_{n}:=\mathbb{P}\left(G_{n}=0\right)$. Temos que os eventos $\left\{G_{n}=0\right\}_{n \geq 0}$ são crescentes, pois

$$
\left\{G_{0}=0\right\} \subset\left\{G_{1}=0\right\} \subset \ldots \subset\left\{G_{n}=0\right\} \subset\left\{G_{n+1}=0\right\} \subset \ldots \subset\{\text { extinção }\}
$$

Isto é,

$$
\bigcup_{n=1}^{\infty}\left\{G_{n}=0\right\}=\{\text { extinção }\}
$$

e, portanto,

$$
\alpha=\mathbb{P}(\text { extinção })=\lim _{n \rightarrow \infty} \mathbb{P}\left(G_{n}=0\right)=\lim _{n \rightarrow \infty} \alpha_{n} .
$$

Além disso, temos que $\gamma(s)$ é contínua, estritamente crescente e convexa em $(0,1)$. Então,

$$
\alpha=\lim _{n \rightarrow \infty} \mathbb{P}\left(G_{n}=0\right)=\lim _{n \rightarrow \infty} \gamma_{n}(0)=\lim _{n \rightarrow \infty} \gamma\left(\gamma_{n-1}(0)\right)=\gamma\left(\lim _{n \rightarrow \infty} \gamma_{n-1}(0)\right)=\gamma(\alpha)
$$

Onde a quarta igualdade de B.7 é obtida usando a continuidade de $\gamma$. Assim, temos que $\alpha$ é a solução de

$$
\gamma(x)=x
$$


em $[0,1]$. Observe que $x=1$ sempre é solução de B.8 e que

$$
\gamma^{\prime}(1)=\lim _{n \rightarrow \infty} \gamma^{\prime}(s)=\lim _{n \rightarrow \infty} \sum_{k=0}^{\infty} i p_{i} s^{i=1}=\sum_{k=0}^{\infty} i p_{i}=\mathbb{E}[X]=m
$$

Uma vez que $\gamma^{\prime}(s)>0$ para $0<s<1$, temos que $\gamma$ é estritamente crescente em $[0,1]$. Além disso, perceba que $\gamma^{\prime \prime}(s)>0 \forall s \in(0,1)$, ou seja, $\gamma$ é convexa em [0,1]. Assim, a figura abaixo representa as duas possibilidades que temos de representação da curva $\gamma$, uma vez que $\gamma(0)>0$.
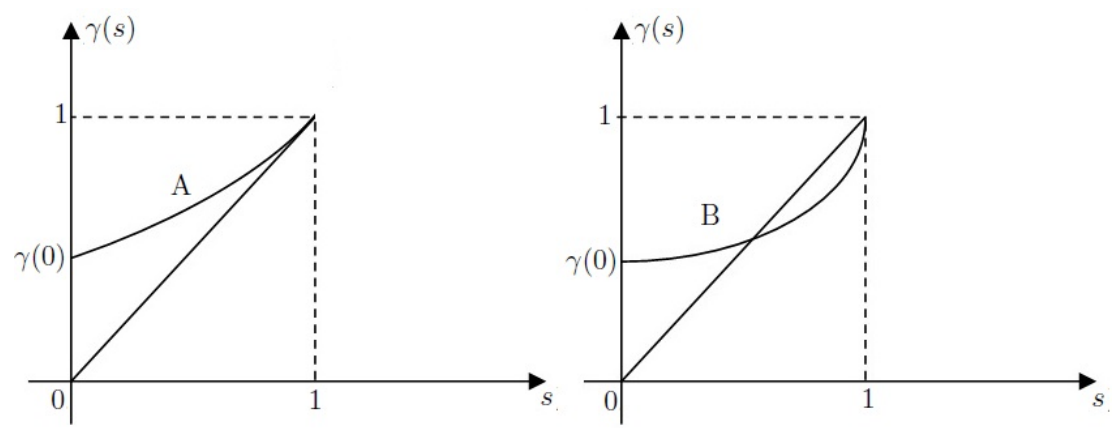

Figura B.1: Função geradora do número de filhos $(\gamma)$ nos casos em que $m \leq 1$ e $m>1$, respectivamente.

Assim, se $m \leq 1$ temos a curva A e a equação B.8 tem uma única solução em $s=1$. Ou seja,

$$
\alpha=\mathbb{P}(\text { extinção })=1 \text {. }
$$

Mas, se $m>1$, perceba que o gráfico de $\gamma$ é representado pela curva B. que intersecta a reta identidade em um ponto $\alpha^{*}<1$. Como $\alpha_{n}$ converge para $\alpha$ quando $\mathrm{n}$ cresce, temos que $\alpha=\alpha^{*}<1$. Isto é,

$$
\alpha=\mathbb{P}(\text { extinção })<1 \text {. }
$$




\section{Apêndice C}

\section{Equações, proposição e lema auxiliares}

Ao longo de todo o texto usamos alguns resultados que podem ser facilmente encontrados em livros. No entanto, com o intuito de facilitar a leitura, apresentamos aqui alguns destes resultados. No capítulo 2, usamos equações encontradas em Prudnikov et al. (2002) e em Gradshteyn e Ryzhik (2007), na prova do teorema 3.1.1 usamos um resultado clássico de cadeias de nascimento e morte e na prova do lema (3.1.1) da seção (3.1.2) usamos um lema para auxiliar a demonstração.

\section{C.1 Equação 5.2.13.9 Prudnikov et al. (2002)}

Seja $x$ tal que $|x|<1 / 4$, então

$$
\sum_{k=0}^{+\infty} \frac{(2 k) !}{k !(k+1) !} x^{k+1}=\frac{1}{2}\left[1-(1-4 x)^{1 / 2}\right] .
$$

\section{C.2 Equação 7.512.11 Gradshteyn e Ryzhik (2007)}

Temos que

$\int_{0}^{1}(1-x)^{\mu-1} x_{p}^{\nu-1} F_{q}\left(a_{1}, \ldots, a_{p} ; \nu, b_{2}, \ldots, b_{q} ; a x\right) d x=\frac{\Gamma(\mu) \Gamma(\nu)}{\Gamma(\mu+\nu)}{ }_{p} F_{q}\left(a_{1}, \ldots, a_{p} ; \nu+\mu, b_{2}, \ldots, b_{q} ; a\right)$

com $\operatorname{Re}(\mu)>0, \operatorname{Re}(\nu)>0, p \leq q+1$. Se $p=q+1$, então $|a|<1$.

Observe que na prova (2.2) usamos a equação acima com $\mu=1, a=4 p q, a_{1}=\frac{m}{2}$, $a_{2}=\frac{m+1}{2}, p=2, q=1$ e $\nu=m+1$. 


\section{C.3 Proposição III 4.1 de Schinazi (1999)}

Seja $\left(X_{n}\right)_{n \geq 0}$ uma cadeia de nascimento e morte tal que

$$
p_{j}=p(j, j+1) q_{j}=p(j, j-1) r_{j}=p(j, j),
$$

com $q_{0}=0$. Tal cadeia será recorrente se, e somente se,

$$
\sum_{j=1}^{\infty} \frac{q_{1} \ldots q_{j}}{p_{1} \ldots p_{j}}=\infty
$$

\section{C.4 Lema auxiliar}

Lema C.4.1. Seja $X_{1}, \ldots, X_{n}$ variáveis aleatórias independentes e identicamente distribuídas com $\mathbb{E}[X]<0$ e $\mathbb{P}(X>0)>0$. Seja $\mathbb{E}\left[e^{u X}\right]=\psi(u)$ finita em alguma vizinhança de $0 e$ defina $\rho=\min _{u>0} \psi(u)$. Então

$$
\lim _{n \rightarrow+\infty} n^{-1} \ln \left(\mathbb{P}\left[\sum_{j=1}^{k} X_{j}>0, k=1, \ldots, n\right]\right)=\ln (\rho) .
$$

Para maiores detalhes, veja o Lema 1 em Aldous e Krebs (1990). 


\section{Referências Bibliográficas}

Abbas et al. (2005) A. K. Abbas, A. H. Lichtman e tradução: C. Reali. Imunologia celular e molecular. Elsevier, Rio de Janeiro. Citado na pág. 1

Aldous e Krebs (1990) D. Aldous e W. Krebs. The birth and assassination process. Statist. Probab. Lett., 10(5):427-430. Citado na pág. 19, 46

Athreya e Ney. (1972) K.B. Athreya e P.E. Ney. Branching processes. Berlin : SpringerVerlag. Citado na pág. 41

Ben-Ari et al. (2011) I. Ben-Ari, A. Matzavinos e A. Roitershtein. On a species survival model. Electronic Communications in Probability, 16:226-233. Citado na pág. 3

Bhattacharya e Waymire (2009) R. N. Bhattacharya e E. C. Waymire. Stochastic Processes With Applications. Society for Industrial \& Applied. Citado na pág. 5, 37, 41

Feller (1968) W. Feller. An Introduction to probability theory and its applications, Vol. 1. Wiley; 3rd edition. Citado na pág. 37

Gradshteyn e Ryzhik (2007) I. S. Gradshteyn e I.M. Ryzhik. Table of integrals, series, and products. seventh edition. Elsevier/Academic Press, Amsterdam. Translated from the Russian, Translation edited and with a preface by Alan Jeffrey and Daniel Zwillinger. Citado na pág. vii, 7,45

Grejo et al. (2016) C. Grejo, F. Machado e A. Roldán-Correa. The fitness of the strongest individual in the subcritical gms model. Electron. Commun. Probab., 21:5 pp. doi: 10. 1214/16-ECP4570. URL http://dx.doi.org/10.1214/16-ECP4570. Citado na pág. 2, 3

Guiol et al. (2011) H. Guiol, F. Machado e R. Schinazi. A stochastic model of evolution. Markov Process. Related Fields, 17(2):253-258. Citado na pág. 1, 2, 3, 4, 9

Guiol et al. (2013) H. Guiol, F. Machado e R.Schinazi. On a link between a species survival time in an evolution model and the bessel distributions. Brazilian Journal of Probability and Statistics, 27(2):201-209. Citado na pág. 3

Harris (1989) T. E. Harris. The theory of branching processes. New York : Dover Publications. Citado na pág. 41 
Luke (1969) Y.L. Luke. The Special Functions and Their Approximations. Vol 1. Academic Press, New York. Citado na pág. 5

Prudnikov et al. (2002) A.P. Prudnikov, Yu.A. Brychkov e O.I. Marichev. Integrals and Series. Volume 1. Elementary Functions. Taylor \& Francis, London. Citado na pág. vii, 7, 45

Ross (2009) S. Ross. Introduction to pobability models. Academic Press; 10Th edition. Citado na pág. 37, 41

Schinazi (1999) R.B. Schinazi. Classical and spatial stochastic process. Birkhäuser Basel. Citado na pág. vii, $12,16,37,46$

Schinazi e Schweinsberg (2008) R.B. Schinazi e J. Schweinsberg. Spatial and non-spatial stochastic models for immune response. Adv. Appl. Prob., 14:255-276. Citado na pág. 2, 9

Skevi e Volkov (2012) M. Skevi e S.E. Volkov. On the generalization of the gms evolutionary model. Markov Process. and Related Fields, 18(2):311-322. Citado na pág. 3 